\title{
Lymphohematopoietic graft-versus-host responses promote mixed chimerism in patients receiving intestinal transplantation
}

\author{
Jianing Fu, ${ }^{1}$ Julien Zuber, ${ }^{1}$ Brittany Shonts, ${ }^{1}$ Aleksandar Obradovic, ${ }^{1}$ Zicheng Wang, ${ }^{2}$ Kristjana Frangaj, ${ }^{1}$ Wenzhao Meng, ${ }^{3}$ \\ Aaron M. Rosenfeld, ${ }^{3}$ Elizabeth E. Waffarn, ${ }^{1}$ Peter Liou, ${ }^{4}$ Sai-ping Lau, ${ }^{1}$ Thomas M. Savage, ${ }^{1}$ Suxiao Yang, ${ }^{1}$ Kortney Rogers, ${ }^{1}$ \\ Nichole M. Danzl, ${ }^{1}$ Shilpa Ravella, ${ }^{5}$ Prakash Satwani, ${ }^{6}$ Alina luga, ${ }^{7}$ Siu-hong Ho, ${ }^{1}$ Adam Griesemer, ${ }^{1,4}$ Yufeng Shen, ${ }^{2}$ \\ Eline T. Luning Prak, ${ }^{3}$ Mercedes Martinez, ${ }^{6}$ Tomoaki Kato, ${ }^{4}$ and Megan Sykes ${ }^{1,4,8}$ \\ 'Columbia Center for Translational Immunology, Department of Medicine and ²Center for Computational Biology and Bioinformatics, Department of Systems Biology, Columbia University, New York, New York, \\ USA. ${ }^{3}$ Department of Pathology and Laboratory Medicine, Perelman School of Medicine, University of Pennsylvania, Philadelphia, Pennsylvania, USA. ${ }^{4}$ Department of Surgery, ${ }^{5}$ Division of Digestive and Liver \\ Diseases, Department of Medicine, ${ }^{6}$ Department of Pediatrics, ${ }^{7}$ Department of Pathology and Cell Biology, and ${ }^{8}$ Department of Microbiology and Immunology, Columbia University, New York, New York, USA.
}

\begin{abstract}
In humans receiving intestinal transplantation (ITX), long-term multilineage blood chimerism often develops. Donor T cell macrochimerism ( $\geq 4 \%$ ) frequently occurs without graft-versus-host disease (GVHD) and is associated with reduced rejection. Here we demonstrate that patients with macrochimerism had high graft-versus-host (GvH) to host-versus-graft (HvG) T cell clonal ratios in their allografts. These $\mathrm{GvH}$ clones entered the circulation, where their peak levels were associated with declines in $\mathrm{HvG}$ clones early after transplant, suggesting that $\mathrm{GvH}$ reactions may contribute to chimerism and control HvG responses without causing GVHD. Consistently, donor-derived T cells, including GvH clones, and CD34+ hematopoietic stem and progenitor cells (HSPCs) were simultaneously detected in the recipients' BM more than $\mathbf{1 0 0}$ days after transplant. Individual GvH clones appeared in ileal mucosa or PBMCs before detection in recipient BM, consistent with an intestinal mucosal origin, where donor $\mathrm{GvH}$-reactive T cells expanded early upon entry of recipient APCs into the graft. These results, combined with cytotoxic single-cell transcriptional profiles of donor T cells in recipient BM, suggest that tissue-resident GvH-reactive donor T cells migrated into the recipient circulation and BM, where they destroyed recipient hematopoietic cells through cytolytic effector functions and promoted engraftment of graft-derived HSPCs that maintain chimerism. These mechanisms suggest an approach to achieving intestinal allograft tolerance.
\end{abstract}

\section{Introduction}

Immune tolerance to avoid the complications of life-long immunosuppression (infections, malignancies, renal failure, cardiovascular disease, and others) could markedly improve quality of life in the field of organ transplantation (1). Induction of hematopoietic chimerism is currently the only approach that has successfully achieved immunological tolerance across MHC barriers in humans (2-5). Tolerance induction protocols achieving sustained full chimerism across HLA barriers likely depend on some level of graft-versus-host $(\mathrm{GvH})$ reactivity that counteracts the hostversus-graft $(\mathrm{HvG})$ response. This is associated with a significant risk of life-threatening graft-versus-host disease (GVHD).

Liver and intestinal allografts have high lymphoid cell loads and the potential to induce GVHD in recipient epithelial tissues (6-8). However, our previous studies in murine models demonstrated that $\mathrm{GvH}$ responses confined to the lymphohematopoietic system, which we termed lymphohematopoietic $\mathrm{GvH}$ responses

Conflict of interest: The authors have declared that no conflict of interest exists. Copyright: @ 2021, American Society for Clinical Investigation.

Submitted: June 25, 2020; Accepted: February 23, 2021; Published: April 15, 2021.

Reference information: J Clin Invest. 2021;131(8):e141698.

https://doi.org/10.1172/JCl141698.
(LGVHRs), can destroy recipient hematopoietic cells without causing GVHD, while still counteracting the HvG response (911). Our recent observations in intestinal transplantation (ITx) further showed that high levels of peripheral blood $\mathrm{T}$ cell mixed chimerism (macrochimerism: $\geq 4 \%$ peak donor $\mathrm{T}$ cells in recipient PBMCs) occur commonly, without GVHD, in recipients of intestinal allografts, and are associated with significantly reduced graft rejection $(P=0.0085)(12,13)$ and slower recipient $\mathrm{T}$ cell repopulation in the graft (14).

ITx has been established as an essential clinical option in the treatment of patients with irreversible intestinal failure who have developed complications associated with the need for parenteral nutrition $(15,16)$. However, the success of ITx is currently limited by high rejection rates, risk of GVHD, and by morbidity and mortality secondary to the high levels of immunosuppression required to overcome the immunogenicity of the graft $(17,18)$. Previous reports suggest that composite allograft transplants, such as multivisceral transplantation (MVTx) and liver-intestinal transplantation (LITx), are associated with reduced rates of intestinal rejection compared with isolated intestinal transplantation (iITx) (19, 20). Our own data have confirmed this observation in association with high peripheral blood chimerism levels in MVTx recipients $(12,13)$. In the current study, we further investigated the under- 
lying mechanisms at both the cellular and the clonotypic levels, providing new insights into the significance of donor $\mathrm{T}$ cell macrochimerism in blood. We hypothesized that outcomes in ITx were largely determined by the exchange of donor and recipient lymphoid tissue and hence the balance of $\mathrm{GvH}$ - and HvG-reactive $\mathrm{T}$ cells. By combining multiparameter flow cytometry, including allele-specific mAbs to distinguish donor- and recipient-derived cells (12-14), with a high-throughput TCR- $\beta$ chain CDR3 sequencing-based approach to track alloreactive $\mathrm{T}$ cells in the $\mathrm{GvH}$ and HvG directions in the graft, circulation, and BM $(14,21)$, we obtained data consistent with this novel mechanism. Single-cell immune profiling of $\mathrm{BM}$ infiltrating donor $\mathrm{T}$ cells revealed a dominant cytotoxic effector phenotype, suggesting a mechanism by which LGVHRs promote donor hematopoietic stem and progenitor cell (HSPC) engraftment in recipient BM.

\section{Results}

The significance of donor $T$ cell macrochimerism in blood and the effect of donor age on the dynamics of recipient $T$ cell replacement in the graft. High-level multilineage donor hematopoietic chimerism was common in recipient blood after ITx, especially in recipients of MVTx, often persisted for longer than 1 year and usually was not associated with clinical GVHD (refs. 12, 13 and Supplemental Figure 1). Clinically significant moderate or severe rejection episodes and de novo development of Class I and Class II donor-specific antibodies (DSAs) up to 600 days after transplant were significantly reduced in patients who showed $\mathrm{T}$ cell macrochimerism $(\geq 4 \%)$ in peripheral blood (Supplemental Figure 1), confirming and extending our previous results $(12,13)$ and strengthening the potential of macrochimerism as a biomarker of clinical outcomes. Blood macrochimerism was also associated with significantly slower replacement of donor graft $\mathrm{T}$ cells by the recipient, not only for CD4 and CD8 $\alpha \beta$ T cells (Figure 1, A-E), but also for $\gamma \delta$ T cells (Figure 1, A, F, and G), which constitute a high proportion of total T cells in both intraepithelial lymphocyte (IEL) (Supplemental Figure 2) and lamina propria lymphocyte (LPL) compartments (Figure 1A), further extending our previous study (14).

As we accumulated more patients and performed additional analysis (Supplemental Tables 1 and 2; Pts 16-24 have been added to the study since our previous publication, ref. 14), we noticed that even in the group of patients with macrochimerism, those whose donors were younger than 1 year old (color-coded by blue) showed significantly faster graft $\mathrm{T}$ cell population by the recipient than those with donor age $\geq 1$ (color-coded by orange), for both CD 4 and CD $8 \alpha \beta$ T cells and to a lesser extent for $\gamma \delta$ T cells (Figure 1, B-G, Supplemental Figure 2).

Donor $T$ cell macrochimerism in blood is associated with enrichment of $\mathrm{GvH}$ over $\mathrm{HvG}$ clones in grafts of patients with donor age 1 year or older and absence of de novo Class I DSAs in circulation. To investigate the dynamic balance of 2-way alloresponses in the intestinal graft, we performed bulk TCR- $\beta$-seq on serial intestinal graft biopsy specimens collected up to 600 days after Tx and tracked the cumulative frequencies of $\mathrm{GvH}$ and $\mathrm{HvG}$ clones within each sample. Given the variable ranges of sample availability over time, differences between the AUC of the GvH and HvG plots $\left(\mathrm{AUC}_{\mathrm{GvH}}-\mathrm{AUC}_{\mathrm{HvG}}\right.$ ) were calculated and normalized by days of measurement (postoperative day $[\mathrm{POD}]_{\text {last }}-\mathrm{POD}_{\text {first }}$ ) (Figure 2,
A-D, Supplemental Table 3). Normalized AUC $>0,=0$, and $<0$ indicate greater, equal, and lower GvH responses, respectively, relative to the $\mathrm{HvG}$ response in the ileum allograft. Patients were categorized into 4 groups based on their status of blood macrochimerism, de novo Class I and Class II DSAs in serum, and donor age (Figure 2E).

The first 2 groups (Figure 2, A and B) included patients with macrochimerism, who were free of de novo Class I DSAs. Group II patients, who had donors with ages younger than 1 year, had overall low levels of GvH and HvG responses in the graft, with normalized AUC values close to 0 . In contrast, group I patients, who had donor age 1 year or older, all had normalized AUC values greater than 0 , and the values were higher in MVTx recipients than in iITx recipients (Figure 2E). The last 2 groups (Figure 2, C and D) included patients without macrochimerism who developed serum Class I and Class II DSAs de novo. Group III patients had donor age 1 year or older and showed higher HvG compared with GvH responses, with normalized AUC values less than 0. Patients in group IV, who had donor age less than 1 year, showed comparable (Pt20) or higher (Pt14) HvG compared with GvH responses. Taken together, donor T cell macrochimerism in blood was associated with enrichment of GvH compared with $\mathrm{HvG}$ clones in the graft of patients with donors 1 year of age or younger and absence of de novo Class I DSAs in circulation. However, the magnitudes of $\mathrm{GvH}$ and $\mathrm{HvG}$ responses were low and values were not correlated with macrochimerism when donors were younger than 1 year of age.

One patient (Pt10) who had high titer (MFI > 10,000) Class I and Class II DSAs in serum prior to transplant that persisted at high levels over a year after Tx (POD 0-377) was not included in the above categorization, and the data for that patient are presented separately (Supplemental Figure 3 and Supplemental Tables 3 and 4). Pt10 (donor age 2 years) was the only MVTx recipient in our cohort who did not develop blood macrochimerism, likely due to the high titer of preformed DSAs that bound to initially circulating donor $\mathrm{T}$ cells and accelerated their clearance, as we previously described (12). This patient had an intermediate rate of recipient $\mathrm{T}$ cell replacement in the ileal graft (Supplemental Figure 3, C and D) and initially had greater GvH compared with HvG T cell clones in the allograft, similar to group I patients.

LGVHRs contribute to donor T cell macrochimerism in blood. We further hypothesized that graft-derived $\mathrm{GvH}$ clones, from intestinal mucosa, gut-associated lymphoid tissues (GALTs), and/or liver, might enter the recipient's peripheral circulation and promote chimerism. Consistent with these hypotheses, higher peak cumulative frequencies of $\mathrm{GvH}$ clones were observed in the blood of patients with macrochimerism compared with those without, regardless of donor age (Figure 3, A and B and Supplemental Table 4). When overlaying the kinetic plots of cumulative frequency of $\mathrm{GvH}$ clones in blood with the percentage of blood $\mathrm{T}$ cell chimerism in each individual patient over time after $\mathrm{Tx}$, a significant difference $(P<0.05$ by Mann-Whitney $U$ test) was seen between patients with $(n=8)$ and without $(n=4)$ macrochimerism (Figure 3C and Supplemental Figure 4). In patients with macrochimerism, the peak of circulating GvH clones either appeared before or simultaneously with the peak of donor $\mathrm{T}$ cell chimerism, whereas in patients without macrochimerism, the much smaller peak of 
A

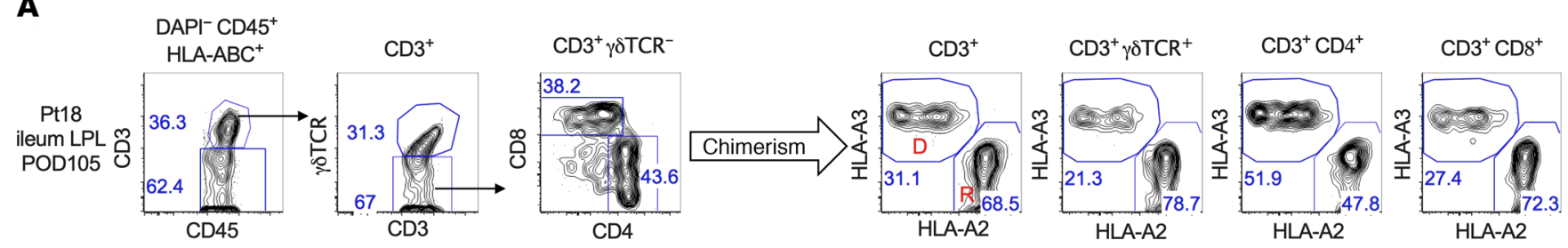

B

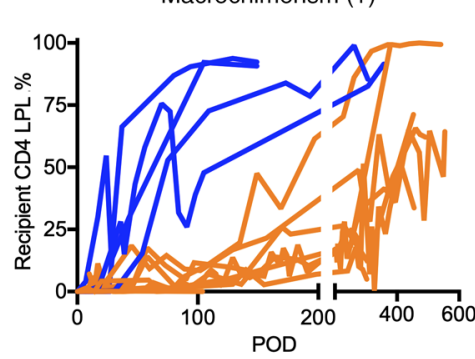

D

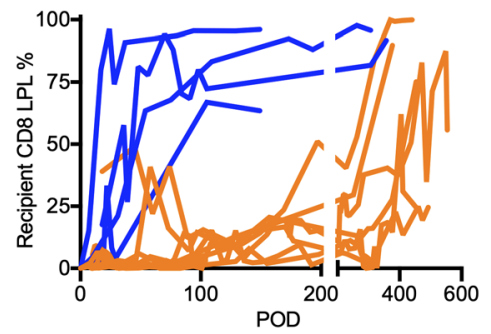

$\mathbf{F}$

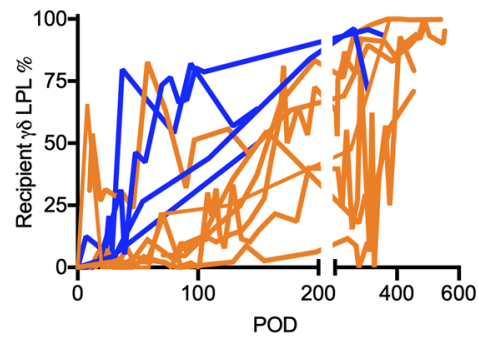

Macrochimerism (-)
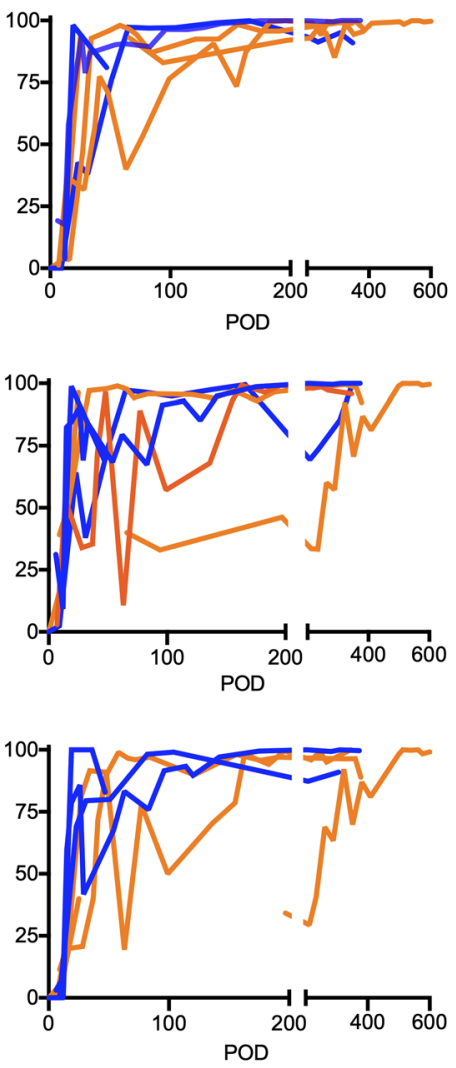

- Donor age $<1$
C

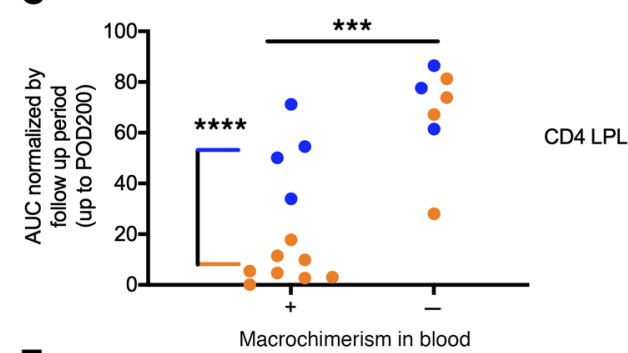

E
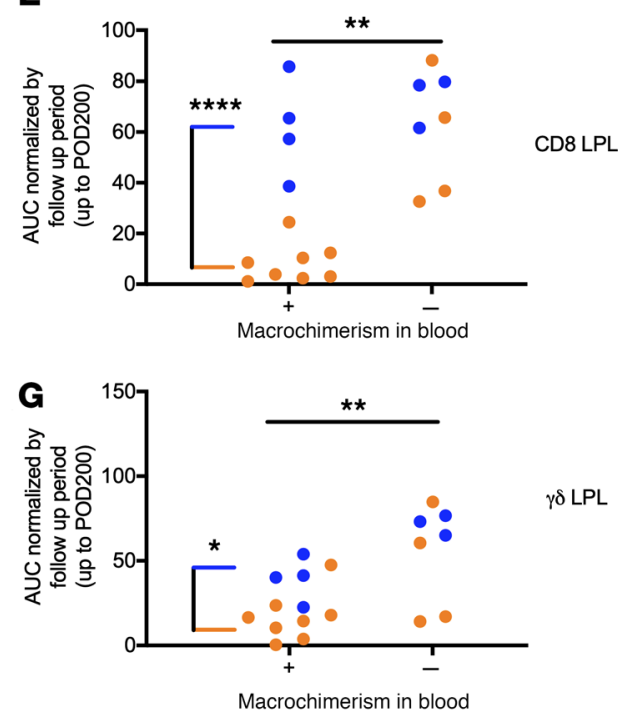

Figure 1. Donor T cell macrochimerism in blood is associated with slower recipient T cell repopulation in the graft after ITx. Among patients with macrochimerism, faster recipient T cell repopulation occurs in grafts with donor age younger than 1 year. (A) Representative flow cytometry (FCM) gating shows percentages of $\gamma \delta, \mathrm{CD}^{+}$, and $\mathrm{CD} 8^{+} \mathrm{T}$ cells and the chimerism among total and subsets of T cells (D, donor; R, recipient) in Pt18 ileum LPLs on POD105. Dynamics of recipient $\mathrm{CD}^{+}(\mathbf{B}), \mathrm{CD}^{+}(\mathbf{D})$ and $\gamma \delta(\mathbf{F})$ T cell repopulation in ileum allograft LPLs in patients with (+, left panel) or without (-, right panel) donor T cell macrochimerism in blood post-ITx (POD0-600). Patients with donor age younger than 1 year and 1 year or older are shown in blue and orange, respectively. AUC of individual patients shown in B, D, and $\mathbf{F}$ normalized by follow-up period (up to POD200) was calculated in $\mathbf{C}, \mathbf{E}$, and $\mathbf{G}$, respectively. Statistical differences were seen between patients with or without macrochimerism, and between patients with donor age younger than 1 year (blue) and 1 year or older (orange) using a 2-tailed unpaired Student's $t$ test; ${ }^{* * *} P<0.0001,{ }^{* * *} P<0.001,{ }^{* *} P<0.01,{ }^{*} P<0.05$.

GvH clones appeared either simultaneously with or much later than the peak of donor $\mathrm{T}$ cell chimerism.

Although $\mathrm{HvG}$ clones were detectable in circulation for over a year after Tx, even in patients with macrochimerism (Supplemental Figure 5), we observed noteworthy declines in the cumulative frequencies of $\mathrm{HvG}$ clones in blood within 40 days after Tx in $4 \mathrm{MVTx}$ patients with macrochimerism (Pts 15, 16", 22, and 23) (Figure 3D) who had particularly high early peak cumulative frequencies of circulating HvG and GvH clones (Figure 3E). In fact, peak cumulative frequencies of $\mathrm{HvG}$ and $\mathrm{GvH}$ clones in the circulation were correla- ted and higher peak levels of $\mathrm{GvH}$ and $\mathrm{HvG}$ clones were associated with a greater early decline of $\mathrm{HvG}$ clones in the blood of patients with macrochimerism (Figure 3, E and F). The data collectively suggest that graft-derived GvH-reactive clones mediate a LGVHR (9-11) that counteracts the systemic $\mathrm{HvG}$ response with minimal to no clinical GVHD, while promoting blood macrochimerism.

We have previously shown that the majority of donor CD8 IELs carried in intestinal grafts are $\mathrm{CD} 69^{+} \mathrm{CD} 103^{+}$tissue resident memory T cells (TRMs) that express lower levels of CD28 compared with circulating $\mathrm{T}$ cells (14). However, a higher 
A
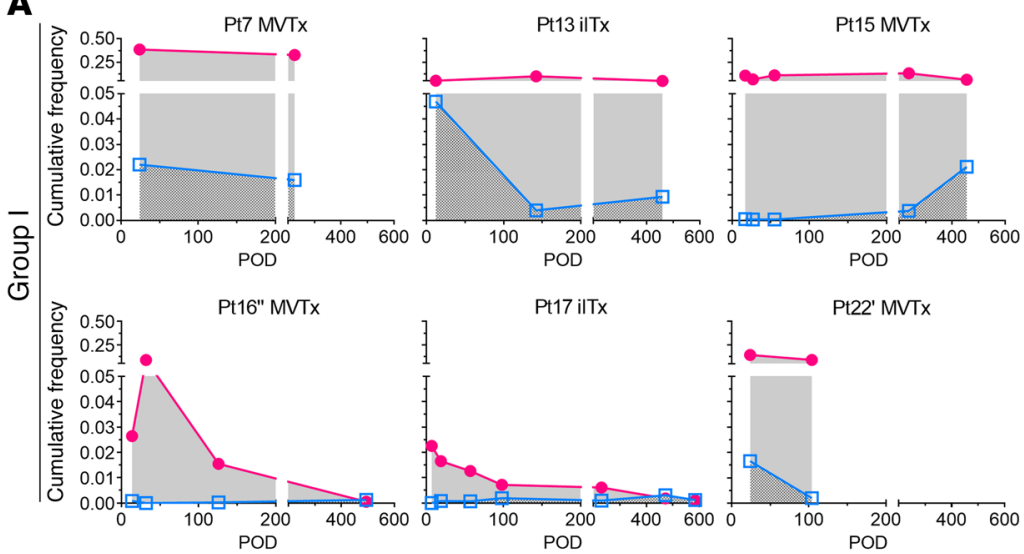

C
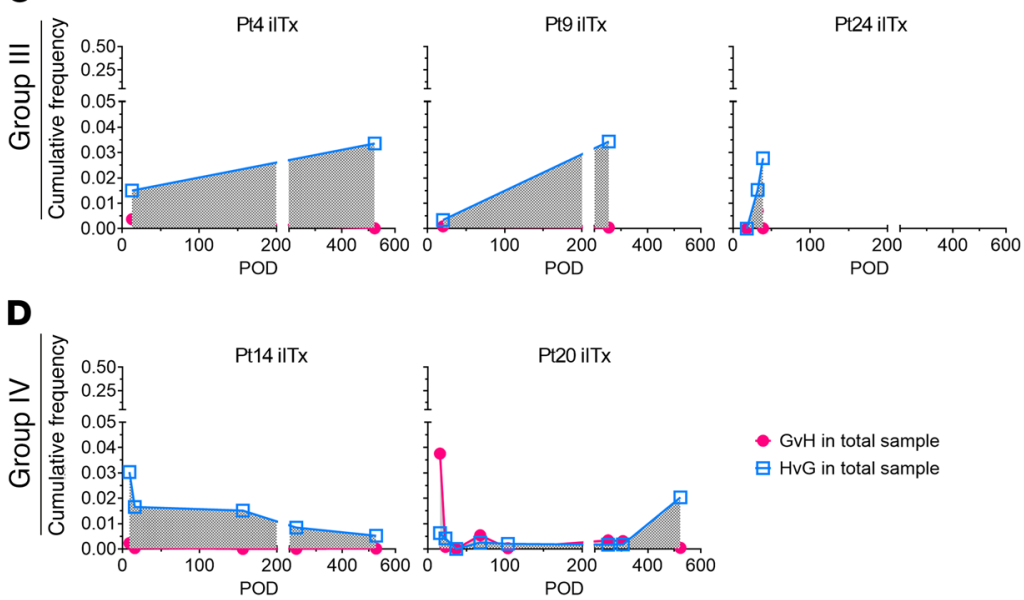

- GvH in total sample 曰 HvG in total sample
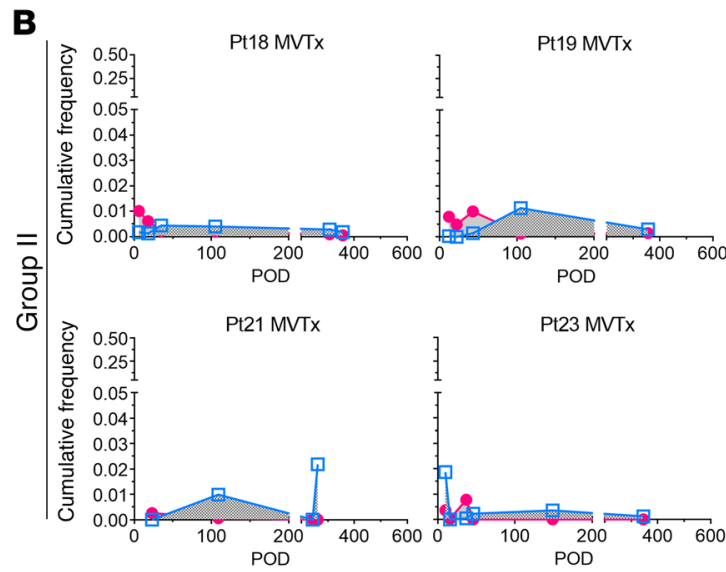

E
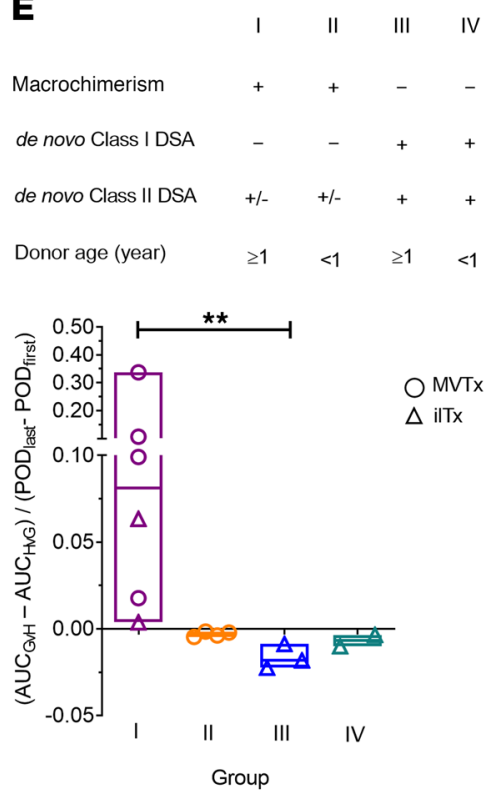

Figure 2. Enrichment of GvH compared with HvG clones in graft and absence of Class I DSAs in circulation are associated with donor T cell macrochimerism. Cumulative frequency of GvH (pink) and HvG (blue) clones among ileum biopsies at indicated time points (A-D). Patients were categorized into 4 groups (I, II, III, IV) based on the presence (+) or absence (-) of donor T cell macrochimerism in blood, de novo Class I and Class II DSAs in serum, and donor age listed in E. (A) Group I patients (Pt7, Pt13, Pt15, Pt16", Pt17, and Pt22) had macrochimerism, were de novo Class I DSA', de novo Class II DSA ${ }^{+-}$, and had donor age of 1 year or older. Pt16' and Pt16" represent the first (LITX), and second (MVTX) transplant in this patient, respectively. (B) Group II patients (Pt18, Pt19, Pt21, and Pt23) had macrochimerism, were de novo Class I DSA', de novo Class II DSA ${ }^{+-}$, and had donor age younger than 1 year. (C) Group III patients (Pt4, Pt9, and Pt24) did not have macrochimerism, developed de novo Class I and Class II DSAs, and had donor age of 1 year or older. (D) Group IV patients (Pt14 and Pt20) did not have macrochimerism, developed de novo Class I and Class II DSAs, and had donor age younger than 1 year. (E) Difference of the areas under the $\mathrm{GvH}$ and $\mathrm{HvG}$ curves $\left(\mathrm{AUC}_{\mathrm{CvH}}-\mathrm{AUC}_{\mathrm{HvC}}\right.$ ) was normalized by days of measurement $\left(\mathrm{POD}_{\text {last }}-\mathrm{POD}_{\text {first }}\right.$ ) of individual patients in groups I to IV and shown by floating bars plots, which include 3 key values: upper extreme, median, and lower extreme. Normalized AUC $>0,=0$, and $<0$ indicate greater, equal, and lower $\mathrm{GvH}$ over HvG responses in the allograft, respectively. Kruskal-Wallis test was performed for multiple comparisons between each pair of groups. Significant difference was seen when comparing group I to group III. ${ }^{* *} P<0.01$.

peak level (>40\%) of CD28 expression was detected on donor $\mathrm{CD} 69^{+} \mathrm{CD} 103^{+} \mathrm{CD} 8$ IELs in patients who had blood T cell macrochimerism than in those without macrochimerism (Supplemental Figure 6), suggesting that some donor TRM cells carried in the intestinal allograft may acquire a transitional phenotype when translocating from graft to circulation. Donor CD8 TRMs in the IELs included effector memory-like (Tem: CD45RA $\mathrm{CCR7}^{-}$) and central memory-like (Tcm: CD45RA-CCR7 ${ }^{+}$) phenotypes (Supplemental Figure 6).

Graft-derived GvH-reactive T cells and HSPCs enter recipient $B M$. We recently demonstrated the presence of functional donor-derived HSPCs in human intestinal allografts and obtained evidence that these contribute to long-term multilineage (T/B/NK/myeloid) blood chimerism, which is frequently observed in MVTx patients (13). We hypothesized that multilineage chimerism in blood after ITx might involve a LGVHR from GvH-reactive donor $\mathrm{T}$ cells migrating from the recipient circulation to the BM, making space for engraftment of HSPCs from the graft. Indeed, graft-derived $\mathrm{CD}^{+} \mathrm{T}$ cells and $\mathrm{CD} 45^{+/ \operatorname{dim}} \mathrm{CD} 34^{+}$ HSPCs (Figure 4A) were simultaneously detected in the BM of 4 of 6 composite graft transplants (Pt16', LITx; Pt16", Pt18, and Pt19, MVTx), and in 1 of 4 iITx recipients (Pt20) (Figure 4B and 
Supplemental Table 5). BM CD34 cell chimerism detected by flow cytometry was confirmed by PCR-based single nucleotide polymorphism (SNP) variant detection (Scisco Genetics) in 2 of 2 patients (Pt16" and Pt19; data not shown).

TCR- $\beta$ CDR3 DNA sequencing identified GvH clones among donor-mappable BM T cells in the 3 patients with donor $\mathrm{T}$ cells in recipient BM. In Pt19, whose BM was sampled twice, the later (POD734) sample demonstrated an increased frequency of $\mathrm{GvH}$ clones, which was associated with a decline in the frequency of HvG clones detected in the same samples. This MVTx recipient was rejection-free through the post-Tx follow-up period (Figure 4C and Supplemental Table 5). Although GvH clones were dominant among donor $\mathrm{T}$ cells in the BM of iTTx recipient Pt24 on POD54 (Figure 4C), there were no detectable donor-derived CD $34^{+}$HSPCs in this sample (Figure 4B and Supplemental Table 5). In iITx recipient Pt20, GvH clones were only detectable in the BM at the late (POD521), but not the early (POD125), time point assayed, and the low CD34 chimerism detected at POD125 disappeared by the later time point (Figure 4, B and C and Supplemental Table 5).

We further tracked individual $\mathrm{GvH}$ clones identified in the $\mathrm{BM}$ to check for their earlier presence in other tissues (Figure 4D, Supplemental Figure 7). In 3 of 6 transplants (Pt19, Pt16", Pt16'; Figure 4D, Supplemental Figure 7), we were able to identify at least $3 \mathrm{GvH}$ clones that preexisted in either the ileum biopsy or PBMCs before their detection in recipient BM. Proportional Venn diagram analysis using all TCR- $\beta$ sequences detected in the ileum biopsy, PBMCs, and BM cells collected on the same day in 3 MVTx recipients (Pt19, Pt18, Pt16”) demonstrate minimal clonal overlap between $\mathrm{BM}$ versus PBMCs $(6.54 \% \pm 7.27 \%)$, which is as low as that for ileum biopsy versus PBMCs $(3.31 \% \pm 3.17 \%)$ (paired $t$ test, $P=0.52$ ), largely excluding potential blood contamination as an explanation for the detection of $\mathrm{GvH}$ clones in the BM (Supplemental Figure 7).

Single-cell transcriptional profiling of BM infiltrating donor $T$ cells revealed dominant clusters of cytotoxic effector $T$ cells with LGVHR potential. Transcriptome level functional gene profiles of FACS-sorted donor $\mathrm{T}$ cells from recipient $\mathrm{BM}$ collected on POD357 of Pt18, POD105 of Pt19, and POD126 of Pt16" were analyzed by $10 \times$ Genomics single-cell RNA sequencing (scRNAseq) to integrate the gene expression (Figure 5, Figure 6, and Supplemental Figure 8) with TCR clonotypes (Figure 5A and Supplemental Figure 9). Minor contaminations of non-T cell populations (CD79A ${ }^{+}$for B cells and CD14 ${ }^{+}$for monocytes) were detected from these patients, as shown in uniform manifold approximation and projections (UMAPs) (Figure 5B) and feature dot plots (Figure 5C). Cluster 7 in Pt18 contains a fraction of T cells and highly expresses several mitochondria-associated genes, and was excluded from further analysis. Major T cell clusters in Pt18 (cluster 0/1/2/3/4/5), Pt19 (cluster 0/1/2), and Pt16" (cluster 0/1/3/4/6) broadly express BM homing markers CXCR4 and ITGA4 (Supplemental Figure $8 \mathrm{~B})$, supporting their migration patterns.

Clusters $0 / 1 / 2 / 3$ in Pt18, cluster 0 in Pt19, and clusters $1 / 3 / 4 / 6$ in Pt16" include mainly CD8 $\alpha \beta$ T cells and $\gamma \delta \mathrm{T}$ cells and share a number of differentially expressed (DE) genes (Supplemental Figure 8C), including highly expressed genes such as KLRB1, DUSP2, GNLY, and CCL4, as shown in the cluster heat- maps (Figure 6A). Gene ontology (GO) term analysis of shared DE genes among clusters $0 / 1 / 2 / 3$ in Pt18, cluster 0 in Pt19, and clusters 1/3/4/6 in Pt16" (Supplemental Figure 8C) identified top relevant biological processes that include cell killing (GO: 0001906), T cell activation (GO:0004210), positive regulation of cytokine production (GO: 0001819), and regulation of immune effector process (GO: 0002697), with several genes highly representative for T cell cytotoxicity (PRF1, GNLY, GZMM, NCR3) and effector T cell (Teff) functions (CD160, FYN, IL7R, CD96; Figure 6B). These observations strongly support the notion of donor graft-derived GvH-reactive T cells entering the circulation and BM, where they attack recipient cells.

Cluster 4 in Pt18, cluster 1 in Pt19, and cluster 0 in Pt16" mainly contained CD 4 and CD8 $\alpha \beta$ T cells and shared many dominant DE genes, such as CCR7, LEF1, SELL, TCF7, and KLF2 (Supplemental Figure 6, A and B and Supplemental Figure 8C), related to undifferentiated/memory stem $\mathrm{T}$ cell subsets, such as stem cell-like memory $\mathrm{T}$ cells $\left(\mathrm{Tscm}, \mathrm{TCF} 7^{+}\right.$), naive $\mathrm{T}$ cells (Tn, $\mathrm{CCR}^{+}$), and central memory $\mathrm{T}$ cells $\left(\mathrm{Tcm}, \mathrm{CCR} 7^{+}\right) . \mathrm{GO}$ term analysis identified biological processes related to not only T cell differentiation (GO: 0030217), lymphocyte proliferation (GO: 0046651), and response to reactive oxygen species (GO: 0000302), but also translational initiation (GO: 0006413) that involves a variety of ribosome family genes, which may indicate active differentiation events ongoing.

TCR clonotype analysis identified several dominant TCR- $\alpha \beta$ clones in FACS-sorted donor T cells from recipient BM of Pt18 POD357 and Pt16" POD126, mainly distributed in cytotoxic Teff clusters (Figures 5 and 6 and Supplemental Figure 9A). Among FACS-sorted donor T cells from recipient BM of Pt19 POD105, a diverse TCR repertoire was identified, with 5 clones having 2 copies and the remaining clones having only 1 copy (Supplemental Figure 9A). Due to the limited number of productive TCR- $\alpha \beta$ sequences identified from each patient by scRNA-seq (Pt18: 1598; Pt19: 382; Pt16": 884), and limited cumulative frequencies of GvH clones identifiable in these donor $\mathrm{T}$ cell-enriched $\mathrm{BM}$ samples by TCR- $\beta$ bulk DNA-seq (Pt18: 0.14\%; Pt19: 0.39\%; Pt16”: 1.66\%), the estimated number of GvH-reactive T cells we could identify by scRNA-seq was less than 3 for Pt18, less than 2 for Pt19, and less than 14 for Pt16". In fact, we identified $1 \mathrm{CD} 4 \mathrm{GvH} \mathrm{T}$ cell in Pt18, 0 GvH T cells in Pt19, and 8 CD8 GvH T cells in Pt16", consistent with the bulk sequencing data. Clonotypes 1, 2, and 3 in Pt18 took up $2.84 \%, 1.53 \%$, and $0.74 \%$ of the TCR- $\alpha \beta$ repertoire identified by scRNA-seq, respectively, and were detected in multiple tissues late after Tx (POD > 300) by high throughput TCR- $\beta$ CDR3 DNA-seq, including the allograft (stomach, duodenum, ileum, colon), native colon, blood, and BM (Supplemental Figure 9B). Clone frequencies of these top 3 clones in Pt18 were much higher in FACS-sorted donor $\mathrm{HLA}^{+} \mathrm{CD} 45^{+}$populations $(>0.001)$ compared with total blood and $\mathrm{BM}(<0.0002)$, further supporting that they are expanded donor TCR- $\alpha \beta$ clones. Similarly, dominant TCR- $\beta$ clones in Pt16" mainly consisted of pre-Tx unmappable clones that were detectable in multiple tissues late after Tx (data not shown). However, we did identify 3 unique TCR- $\beta$ clones in Pt16" POD126 BM that were identifiable as GvH-reactive CD8 clones (Supplemental Figure 9C), including 1 dominant clone (clonotype 7) with 6 copies and 2 other clones (clonotype 220, 470) 
A

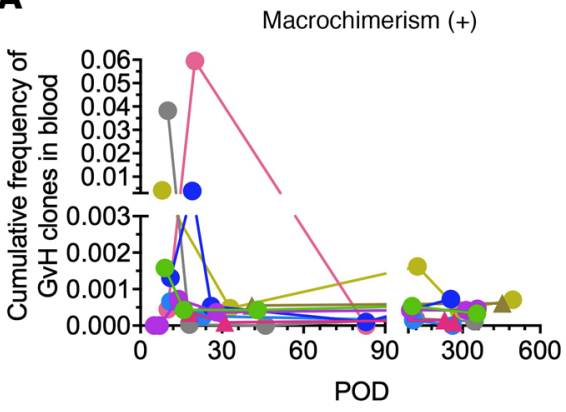

Macrochimerism (-)

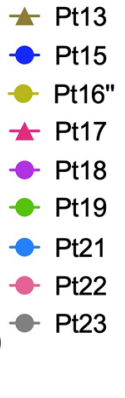

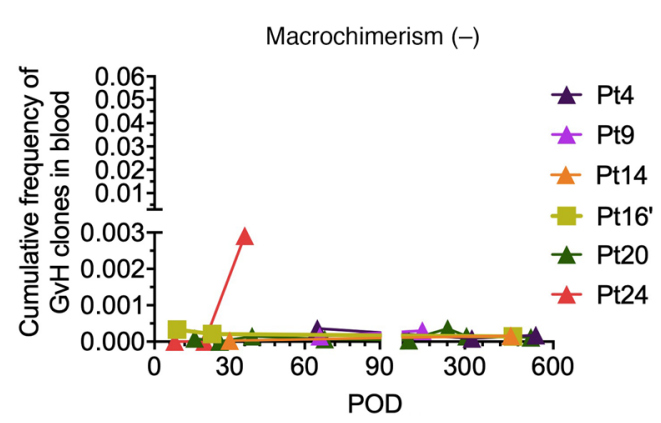

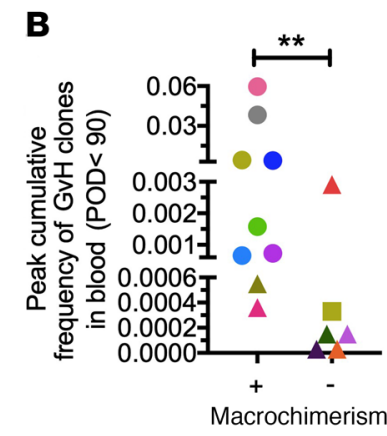

C
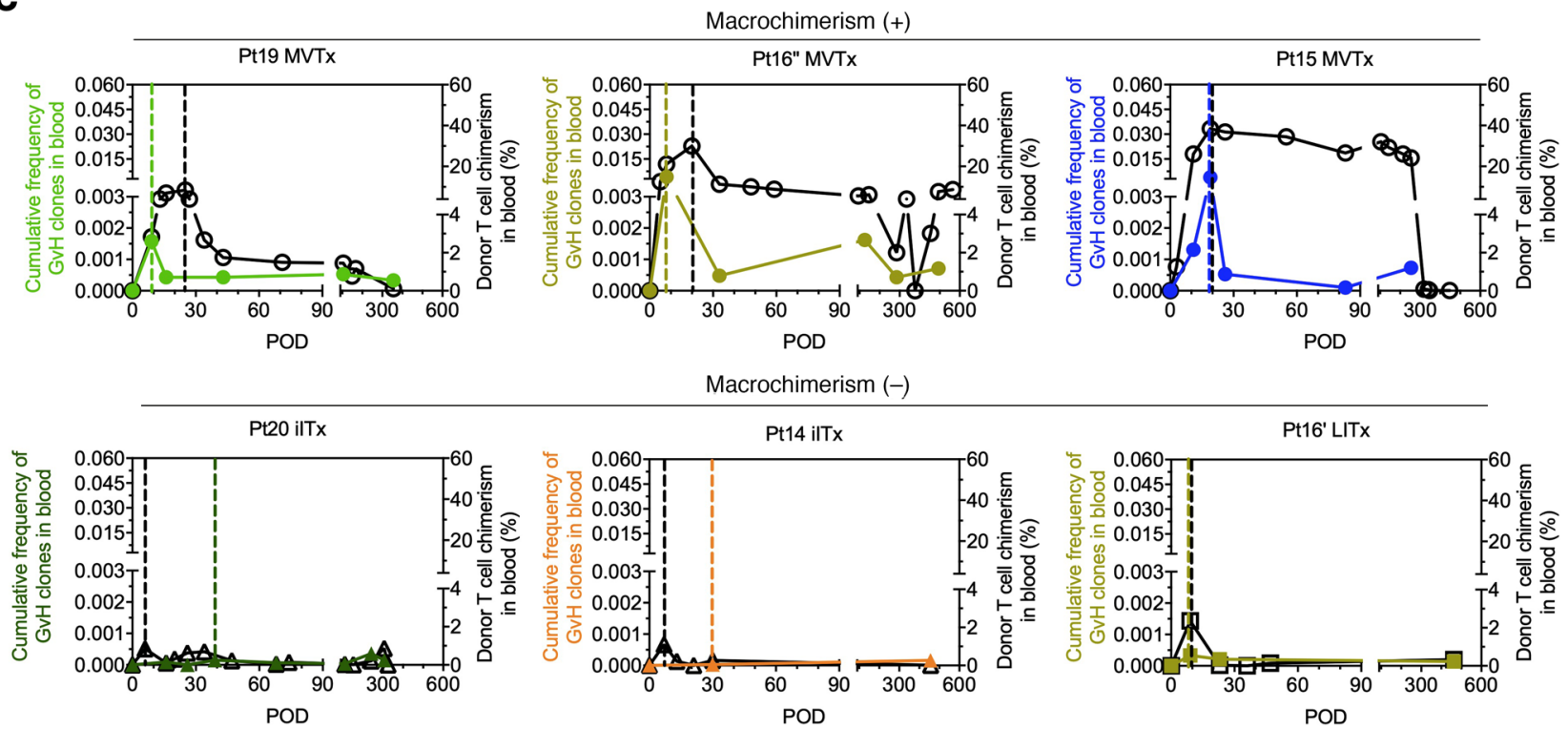

Macrochimerism (-)
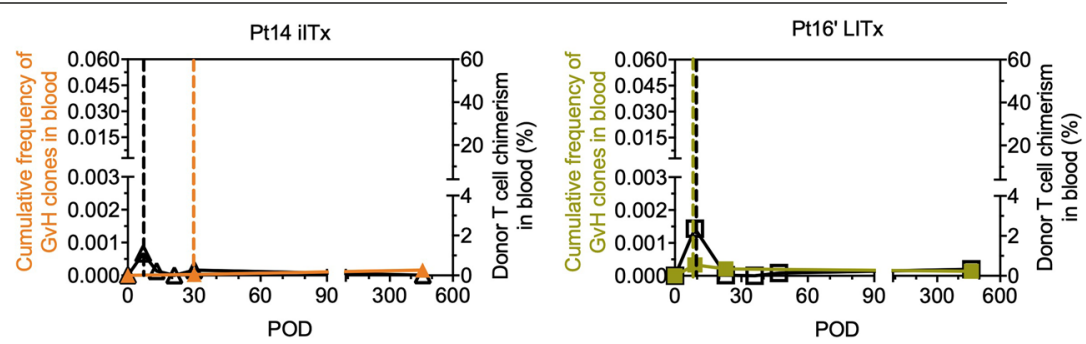

D
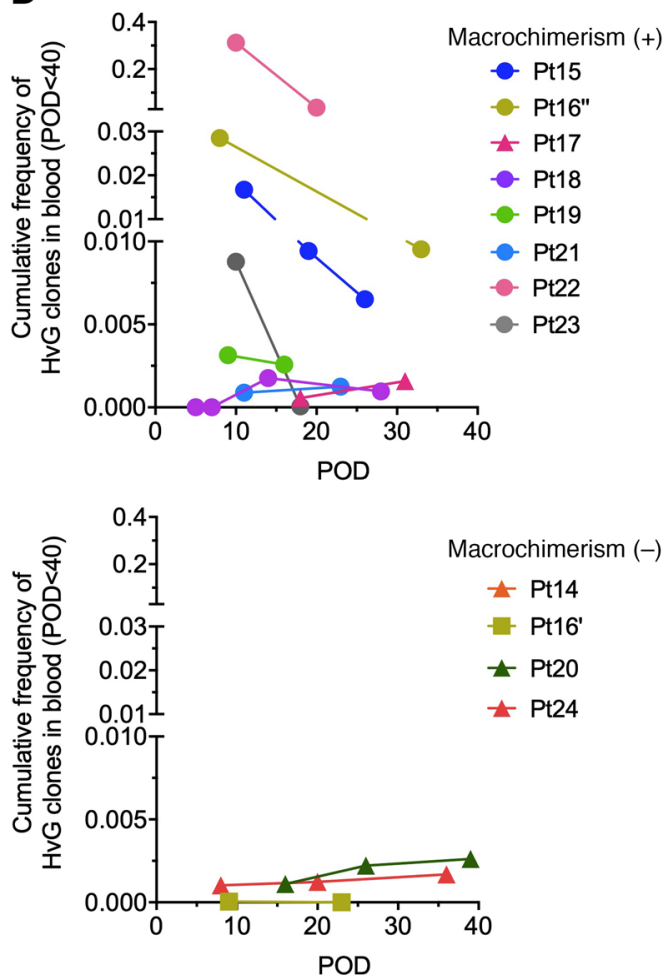

E
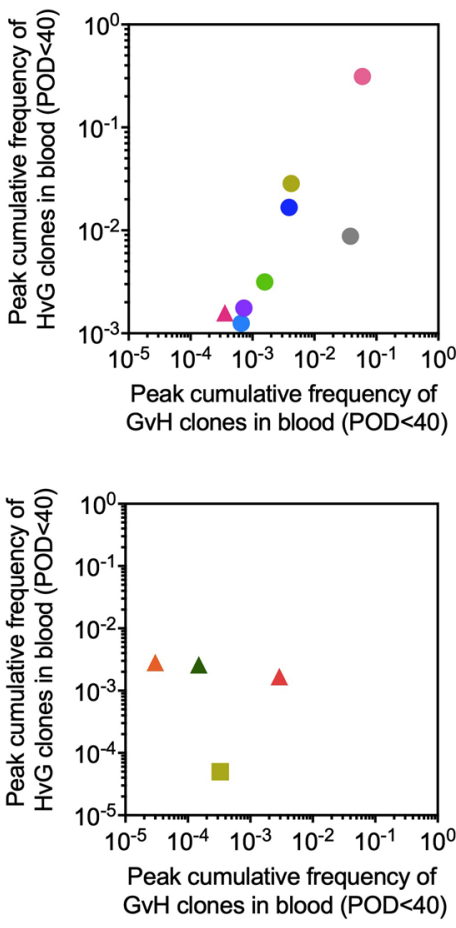

F

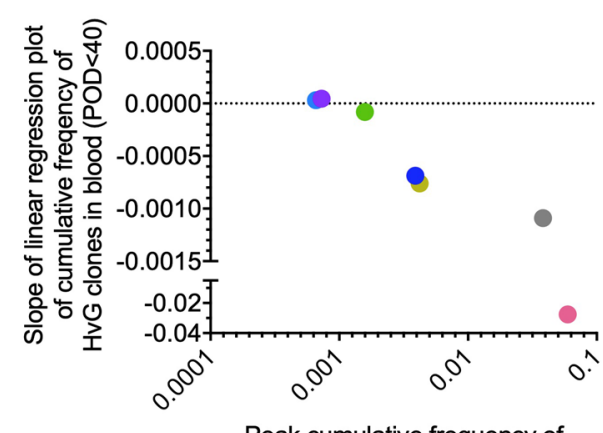

Peak cumulative frequency of GvH clones in blood (POD<40)

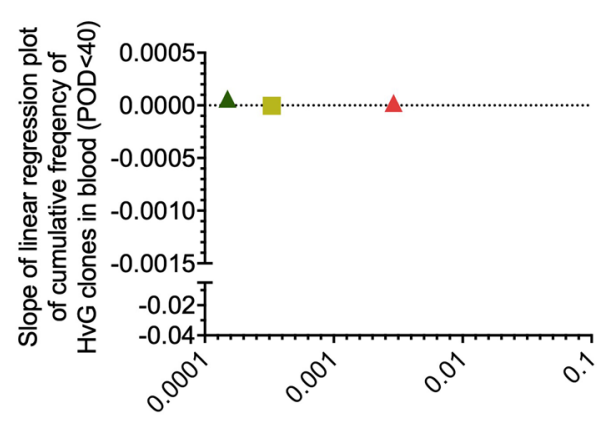

Peak cumulative frequency of GvH clones in blood (POD<40) 
Figure 3. Lymphohematopoietic GvH responses contribute to donor T cell macrochimerism in blood. (A) Kinetics of cumulative frequency of $\mathrm{GvH}$ clones in the circulation of patients with (+) or without (-) donor T cell macrochimerism in recipients of MVTx (circles), LITx (squares), or ilTx (triangles). (B) Peak cumulative frequency of GvH clones in blood in patients with or without macrochimerism. ${ }^{* *} P<0.01$, Mann-Whitney $U$ test. (C) Kinetics of cumulative frequency of GvH clones (left $y$ axis: colored curve, solid symbols) and donor T cell chimerism (right $y$ axis: black curve, open symbols) in circulation of representative patients with (+: Pt19, Pt16", and Pt15) or without (-: Pt20, Pt14, and Pt16') T cell macrochimerism. Additional patients are shown in Supplemental Figure 4. Patients with at least one time point of TCR-seq data and 2 time points of FCM chimerism data within 40 days after Tx were included in this analysis. Colored dotted vertical line indicates the POD of peak cumulative frequency of circulating $\mathrm{GvH}$ clones and black dotted vertical line indicates the POD of peak donor $T$ cell chimerism in blood. (D) Kinetics of cumulative frequency of HvG clones in the circulation of patients with or without donor T cell macrochimerism who were sequenced on at least 2 time points within 40 days after Tx. (E) Correlation of peak cumulative frequency of GvH ( $x$ axis) and HvG clones ( $y$ axis) in blood within 40 days after Tx in patients with or without macrochimerism. (F) Association of peak cumulative frequency of GvH clones in blood and slope of linear regression plot of cumulative frequency of HvG clones in blood within 40 days after Tx in patients with or without macrochimerism. Increased slope absolute value indicates increased rate of change in cumulative frequency of circulating HvG clones.

each with 1 copy (Supplemental Figure 9C). BM-infiltrating donor CD8 GvH-reactive T cells showed cytotoxic Teff transcriptional profiles (TBX21 $1^{+}, \mathrm{GZMB}^{+}, \mathrm{PRF}^{+}, \mathrm{GZMA}^{+}$, and $\mathrm{GNLY}^{+}$), suggesting a mechanism for destruction of host cells. These $\mathrm{CD} 8 \mathrm{GvH}$ clones are contrasted with a different cluster of donor CD8 Tscm cells that showed stem cell-like features $\left(\mathrm{TCF}^{+}, \mathrm{LEF}^{+}, \mathrm{SELL}^{+}\right.$, and $\mathrm{CCR7}^{+}$) in Figure $6 \mathrm{C}$. These $3 \mathrm{GvH} \mathrm{CD} 8$ clones were detectable in the ileal allograft as early as 14 days after Tx and 2 of 3 clones were broadly distributed in the stomach, duodenum, ileum, peripheral blood, and BM on POD126. These clones persisted in late PBMCs and/or lymph nodes on POD494 (Supplemental Figure 9C).

To test the possibility that donor regulatory $\mathrm{T}$ cells (Tregs) control HvG reactivity in the stem cell niche and allow engraftment of graft-derived HSPCs in the recipient BM $(13,22,23)$, we analyzed Foxp $3^{+} \mathrm{T}$ cells among CD4 clusters identified in our scRNA-seq study from Pt18, Pt19, and Pt16" (Supplemental Figure 10). While only 2 and 4 Foxp $3^{+}$Tregs were identified in clusters $4 / 5$ of Pt18 and cluster 1 of Pt19, respectively, we did identify 35 Foxp $^{+}$Tregs in BM of Pt16" that mainly distributed in cluster O (Supplemental Figure 10A). BM-infiltrating donor CD4 Tregs showed stem cell-like features $\left(\mathrm{TCF}^{+} \mathrm{LEF}^{\mathrm{hi}}\right.$ SELL ${ }^{\text {hi; }}$ S Supplemental Figure 10B). The frequency of donor Tregs in recipient BM detected by scRNA-seq was consistent with that detected by flow cytometry (Supplemental Figure 10C). Thus, although further studies are needed, BM-infiltrating donor Tregs might contribute to the engraftment of donor HSPCs.

\section{Discussion}

We demonstrate here that expansion of GvH-reactive donor $\mathrm{T}$ cells, likely triggered by early infiltration of recipient myeloid cells into the allograft mucosa (14), relative to HvG-reactive recipient $\mathrm{T}$ cells entering the graft mucosa, is associated with peripheral blood macrochimerism. These GvH-reactive donor T cells appear early in the recipient's circulation in association with rapid reduc- tions in circulating $\mathrm{HvG}$ clones. Patients demonstrating these phenomena have reduced rejection rates and reduced de novo DSA production compared with those who do not, and tend to be recipients of MVTx rather than iITx. The larger GvH T cell load carried with MVTx compared with iITx, combined with the removal of more recipient lymphocytes in tissues such as the spleen, favors the GVHR in MVTx recipients. Remarkably, by integrating T cell clonotype, alloreactivity, and functional gene profiles, we have demonstrated that this GVHR migrates into the recipient BM along with donor HPSCs, which we have previously shown to be carried in intestinal allografts (13). Many of the donor T cells in recipient BM have cytotoxic transcriptional profiles, including those that are identifiable as GvH CD8 clones. Overall, our findings are consistent with a paradigm wherein LGVHR promotes donor hematopoietic engraftment in the blood and marrow by attacking recipient hematopoietic cells in the $\mathrm{BM}$, making space for donor HPSC engraftment and controlling HvG reactivity, as previously shown only in rodent studies (9-11). Similar to results in rodents not receiving recent chemotherapy or radiation therapy (11), LGVHR in these patients was not associated with GVHD and therefore is a GvH reaction that does not migrate into the epithelial GVHD target organs. Murine models showed that the presence of inflammation within an epithelial tissue is a prerequisite for the trafficking of activated GvH T cells to those sites (11). The lack of GVHD may therefore reflect a lack of inflammation in skin, lung, or native colon of MVTx patients with blood macrochimerism. This, to our knowledge, is the first direct demonstration of LGVHR in humans. An important therapeutic implication is that chimerism might be augmented and rendered permanent by the infusion of donor BM CD34 $4^{+}$HSPCs at the time of peak LGVHR. While previous attempts $(24,25)$ at BM augmentation with ITx failed to show significant clinical improvement, these infusions were not timed at the peak of LGVHR and in one study the donor graft was irradiated (7.5 Gy), likely reducing the donor lymphoid load and thereby impairing LGVHR.

The absence of de novo Class I DSAs in the recipient circulation after $\mathrm{Tx}$ in patients with macrochimerism likely reflects overall improved control of HvG responses in patients with dominant GvH reactivity. In one highly illustrative case, however, DSAs that were present prior to transplant were shown to bind to initially circulating donor cells in vivo and accelerate their clearance (12). Consequently, this patient (Pt10) was the only MVTx recipient who did not achieve blood chimerism beyond the first week. Interestingly, this patient initially had greater $\mathrm{GvH}$ compared with $\mathrm{HvG}$ responses in the allograft, presumably because GvH-reactive T cells in the graft were protected from circulating DSAs. This result points to the primacy of the expansion of GvH-reactive T cells in the graft mucosa of MVTx recipients.

The relationship between mucosal GvH and HvG reactivity and macrochimerism was clearly observed in grafts from donors older than 1 year of age. In such grafts, it is likely that graftresident TRMs with $\mathrm{GvH}$ cross-reactivity expand, after which some cells leave the tissue of residence, entering the circulation and acquiring the circulating $\mathrm{T}$ cell phenotype (e.g., upregulation of CD28, loss of CD69 and CD103) (13, 14). Exchange between TRMs and $\mathrm{T}$ cells in the circulation and lymphoid organs has recently been reported in mice and humans (26-29). Grafts from 
A
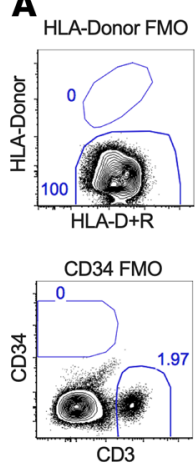

Pt19 BM POD105
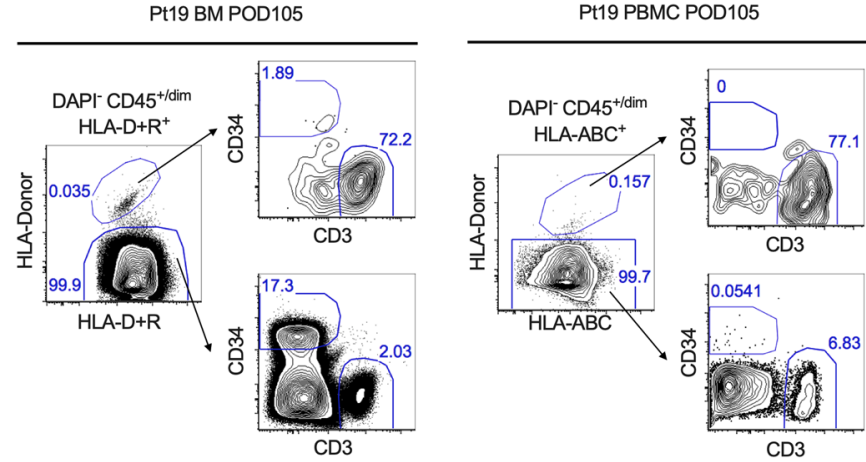

B
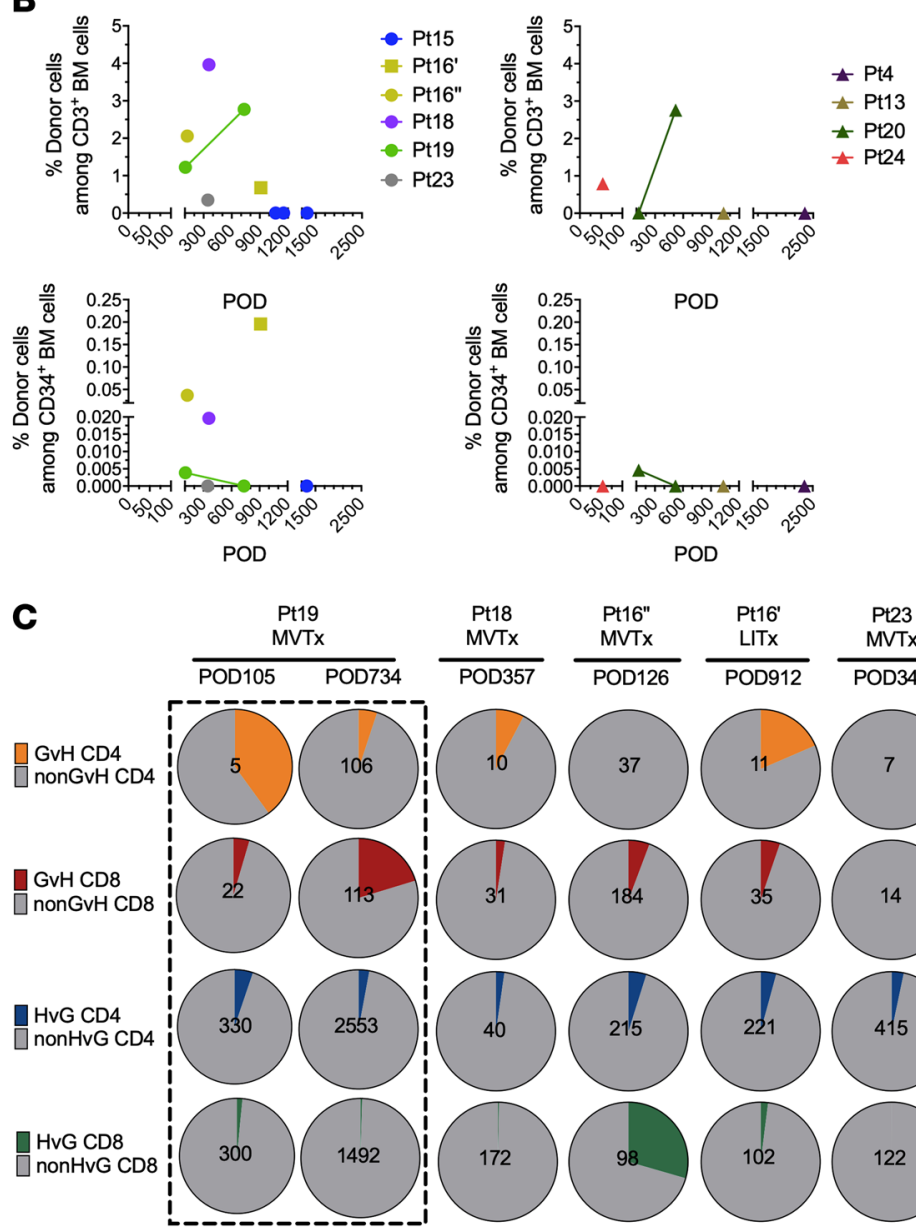

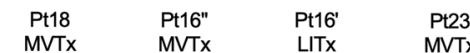
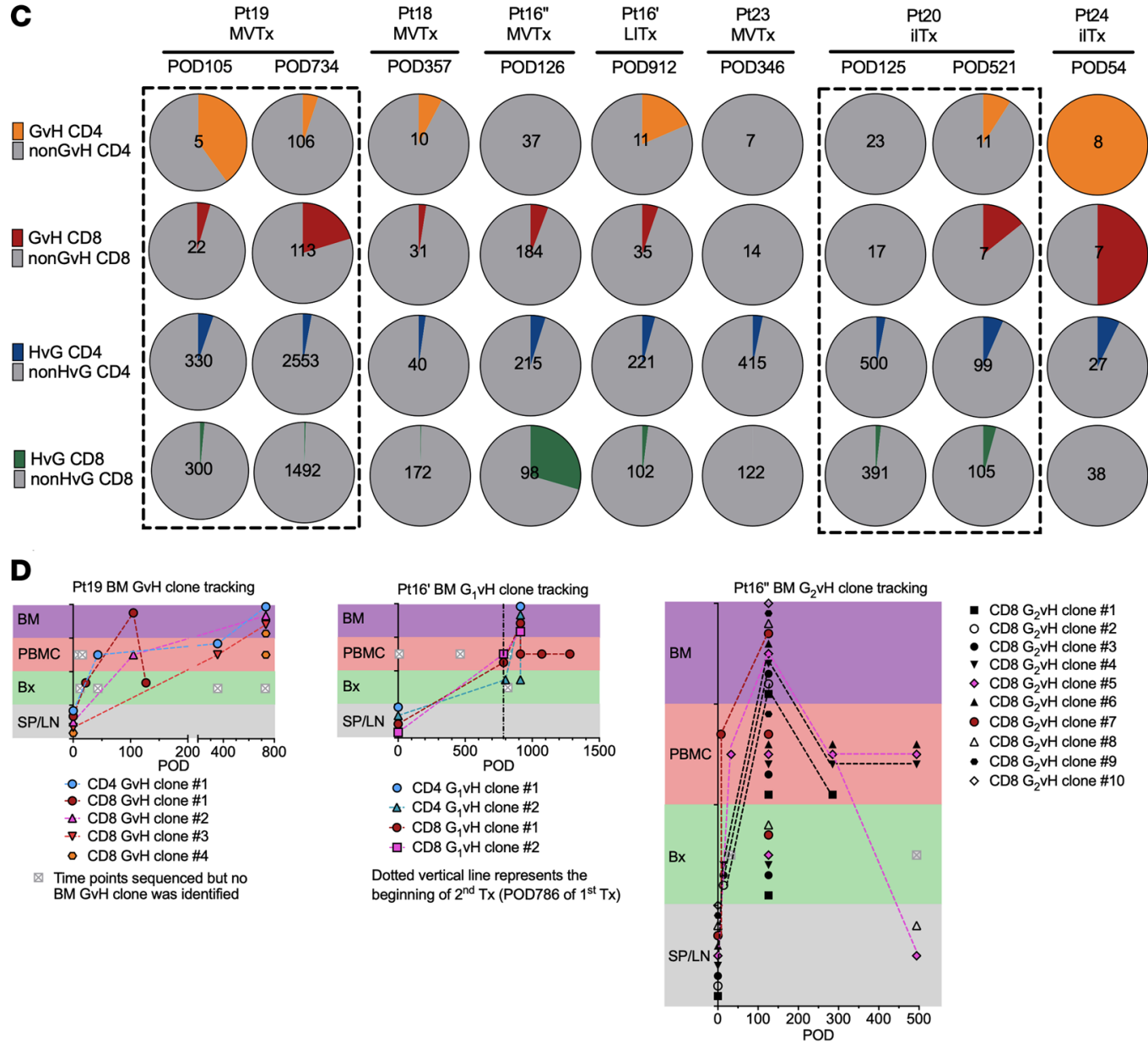

- $\mathrm{CD} 8 \mathrm{G}_{2} \mathrm{vH}$ clone \#

- CD8 $\mathrm{G}_{2} \mathrm{vH}$ clone \#2

- CD8 $\mathrm{G}_{2} \mathrm{vH}$ clone \#3

- CD8 $\mathrm{G}_{2} \mathrm{VH}$ clone \#4

$\diamond \mathrm{CD} 8 \mathrm{G}_{2} \mathrm{vH}$ clone \#5

- CD8 $\mathrm{G}_{2} \mathrm{vH}$ clone \#6

- $\mathrm{CD} 8 \mathrm{G}_{2} \mathrm{vH}$ clone \#

$\triangle \mathrm{CD} 8 \mathrm{G}_{2} \mathrm{vH}$ clone \#8

- CD8 G $\mathrm{vHH}$ clone \#

\& $\mathrm{CD} 8 \mathrm{G}_{2} \mathrm{vH}$ clone \#10 
Figure 4. LGVHR migrates to the bone marrow, making space for engraftment of HPCs from the graft. (A) Flow cytometric analysis of a MVTx recipient (Pt19) BM on day 105 after Tx, showing the presence of donor-derived T cells and CD45+CD34+ HSPCs. Similar gating in Pt19 POD105 PBMCs is shown as a control. Fluorescence minus one (FMO) controls for donor HLA (HLA-A3) and CD34 are shown for Pt19 P0D105 BM. HLA-A2 is expressed by both the donor and recipient cells of Pt19 (HLA-D+R). (B) Donor CD3 ${ }^{+} T$ cells and CD34+ $\mathrm{HSPCs}$ were simultaneously detected in the BM of 4 of 6 transplants receiving composite grafts (LITx: Pt16'; MVTx: Pt16", Pt18, and Pt19) and of 1 of 4 ilTx (Pt20 POD125). Pt16 was retransplanted on POD786 following the first Tx. Pt16' (LITx: first Tx) POD912 was the same day as Pt16" (MVTX: second Tx) POD126, when we collected the BM aspirate and detected cells from both donors. (C) Cumulative frequencies of alloreactive clones detectable in the BM of patients receiving MVTx (Pt19, Pt18, Pt16", Pt23), LITx (Pt16'), and ilTx (Pt20, Pt24) are shown in pie charts, including $\mathrm{CvH}$ CD4 among donor-mappable CD4 sequences, CvH CD8 among donor-mappable CD8 sequences, HvG CD4 among recipient-mappable CD4 sequences, and HvG CD8 among recipient-mappable CD8 sequences. The counts of mappable unique sequences are annotated in each pie chart and summarized in Supplemental Table 5. (D) CD4 and CD8 CvH clones were detected in either the ileum biopsy or PBMCs in addition to recipient BM in Pt19, Pt16', and Pt16".

younger donors ( $<1$ year old) showed very few alloreactive $\mathrm{T}$ cells in either direction, yet populated rapidly with recipient $\mathrm{T}$ cells in the absence of rejection, presumably reflecting the absence of a preexisting mucosal TRM compartment and hence its physiological filling from the circulation. Very young donors have previously been shown to have significantly lower proportions of $\mathrm{CD} 69^{+} \mathrm{CD} 103^{+} \mathrm{CD} 8 \mathrm{~T}$ cells in mucosal sites compared with young adults (30). Nevertheless, recipients of MVTx from these donors also showed macrochimerism and evidence for LGVHR in the blood and BM (e.g., Pt18 and Pt19), suggesting that GvH reactivity may also originate from donor liver or possibly from naive $\mathrm{T}$ cells in lymph nodes carried with the allograft.

Several mechanisms may account for the ability of $\mathrm{GvH}$ reactivity to counteract rejection $(1,31)$ : (a) direct destruction of donor-reactive host $\mathrm{T}$ cells by GvH-reactive donor T cells via cytotoxic mechanisms, as suggested by the cytotoxic transcriptional pattern of identifiable GvH clones; (b) a veto mechanism attributed to activated donor $\mathrm{CD} 8^{+} \mathrm{T}$ cells $(32,33), \gamma \delta \mathrm{T}$ cells $(34)$, and other lymphoid populations $(35,36)$ that is independent of alloantigen recognition (37) can prevent marrow graft rejection by counterattack of host cytotoxic cells, mainly through FasL-mediated activation-induced cell death (38); and (c) $\mathrm{CD} 34^{+}$hematopoietic progenitors may counteract rejection through a deletion-based mechanism mediated by TNF- $\alpha$ (39). The correlation we observed between $\mathrm{GvH}$ reactivity and loss of $\mathrm{HvG}$ clones in the circulation early after $\mathrm{Tx}$, and the transcriptional profiles of BM-infiltrating donor $\mathrm{T}$ cells are consistent with active destruction of $\mathrm{HvG} \mathrm{T}$ cells and host hematopoietic cells by cytotoxic GvH-reactive T cells, i.e., by the LGVHR through production of perforin (PRF1), granzyme (GZMA), and TNF- $\alpha$, to a lesser extent by induction of apoptosis through upregulation of FasL (FASLG) or TRAIL (TNFSF10), under the transcriptional regulation of TBX21, EOMES, RORC, and RUNX3 (Supplemental Figure 8D). We identified several GvH clones among BM-infiltrating donor T cells in 3 of 3 MVTx recipients. In one of these patients, scRNA-seq captured several GvH CD8 clones and transcriptional analysis demonstrated a cytotoxic effector phenotype, consistent with our hypothesis that these clones promote donor HPSC engraftment by creating hematopoietic space by attacking recipient hematopoietic cells. The hematopoietic space created by the LGVHR may enable the survival and expansion of donor HSPCs from the graft that enter the circulation, $\mathrm{BM}$, and thymus, resulting in de novo donor $\mathrm{T}$ cell generation and promoting persistent multilineage chimerism, as observed (13). However, some persistence (POD>200) of HvG T cells in the graft and periphery was observed even in patients with macrochimerism, suggesting that several mechanisms may contribute to the control of $\mathrm{HvG}$ responses.

Tregs showed little to no contribution to the late hyporesponsiveness of post-Tx recipient $\mathrm{T}$ cells to donor antigens in 2 of 2 patients (Pt15, Pt16") tested (Supplemental Figure 11), arguing against a strong systemic role for Tregs in suppressing anti-donor reactivity. In contrast, Tregs at least partially contributed to the peripheral tolerance of donor circulating $\mathrm{T}$ cells to the recipient antigens late after Tx (13). Additionally, BMinfiltrating donor Tregs with stem cell-like features $\left(\mathrm{TCF}^{+}\right.$ LEF $^{\text {hi }}{ }^{\text {SELL }}{ }^{\text {hi) }}$ were detected in our scRNA-seq studies. These Tregs might contribute to the stem cell niche to allow engraftment of graft-derived HSPCs $(13,22,23)$.

$\mathrm{BM}$ in mice and humans is thought to be a reservoir for memory $\mathrm{T}$ cell maintenance (40-42). BM T cells can rapidly acquire effector function and eliminate infected and malignant cells $(41,43)$. They are largely quiescent, are thought to undergo maintenance proliferation in response to BM stromal cytokines (42), and have phenotypic features of TRMs, including CD69 and lack of CD28 on $\mathrm{CD} 8^{+} \mathrm{T}$ cells (44). It is unclear whether the BM is a true niche for long-term residency or whether there are separate niches for resident and nonresident memory T cells (45-47). The scRNA-seq studies we performed indicated that some of these donor T cells, including GvH-reactive T cells, are indeed cytotoxic/effector cells, whereas others have a more memory/stem cell-like transcriptional profile, including both Tregs and non-Tregs. Thus, our data suggest a migration pathway for both effector T cells and TRM cells from an allograft into a recipient's BM. Our data provide, to our knowledge, the first demonstration that human $\mathrm{T}$ cells can migrate from an organ allograft to the recipient's BM.

A large number of donor T cells in recipient $\mathrm{BM}$ were $\gamma \delta \mathrm{T}$ cells, whose function is unknown. Although $\gamma \delta \mathrm{T}$ cells normally account for $1 \%$ to $10 \%$ of circulating $\mathrm{T}$ lymphocytes in humans, they constitute the major subset of resident $\mathrm{T}$ cells in mucosa and skin (48). Transcriptional profiling of BM-infiltrating donor $\gamma \delta \mathrm{T}$ cells demonstrated expression of cytotoxic and effector genes, suggesting a novel migratory pathway from intestinal mucosa and/or GALTs to BM. These cells may contribute to the LGVHR as nonspecific effector cells, as earlier in vitro studies indicate minor, if any, alloreactivity of human $\gamma \delta \mathrm{T}$ cells $(49,50)$. Additional repertoire and functional studies of $\gamma \delta \mathrm{T}$ cells are needed.

In conclusion, our findings suggest that locally expanded GvH-reactive donor $\mathrm{T}$ cells in the graft enter the recipient circulation and attack host hematopoietic cells, leading to early donor $\mathrm{T}$ cell blood chimerism. This LGVHR allows the engraftment of graft-derived HSPCs in the recipient BM, thereby promoting sustained mixed chimerism, as observed (13). Our studies collectively provide insight into the mechanism of durable 

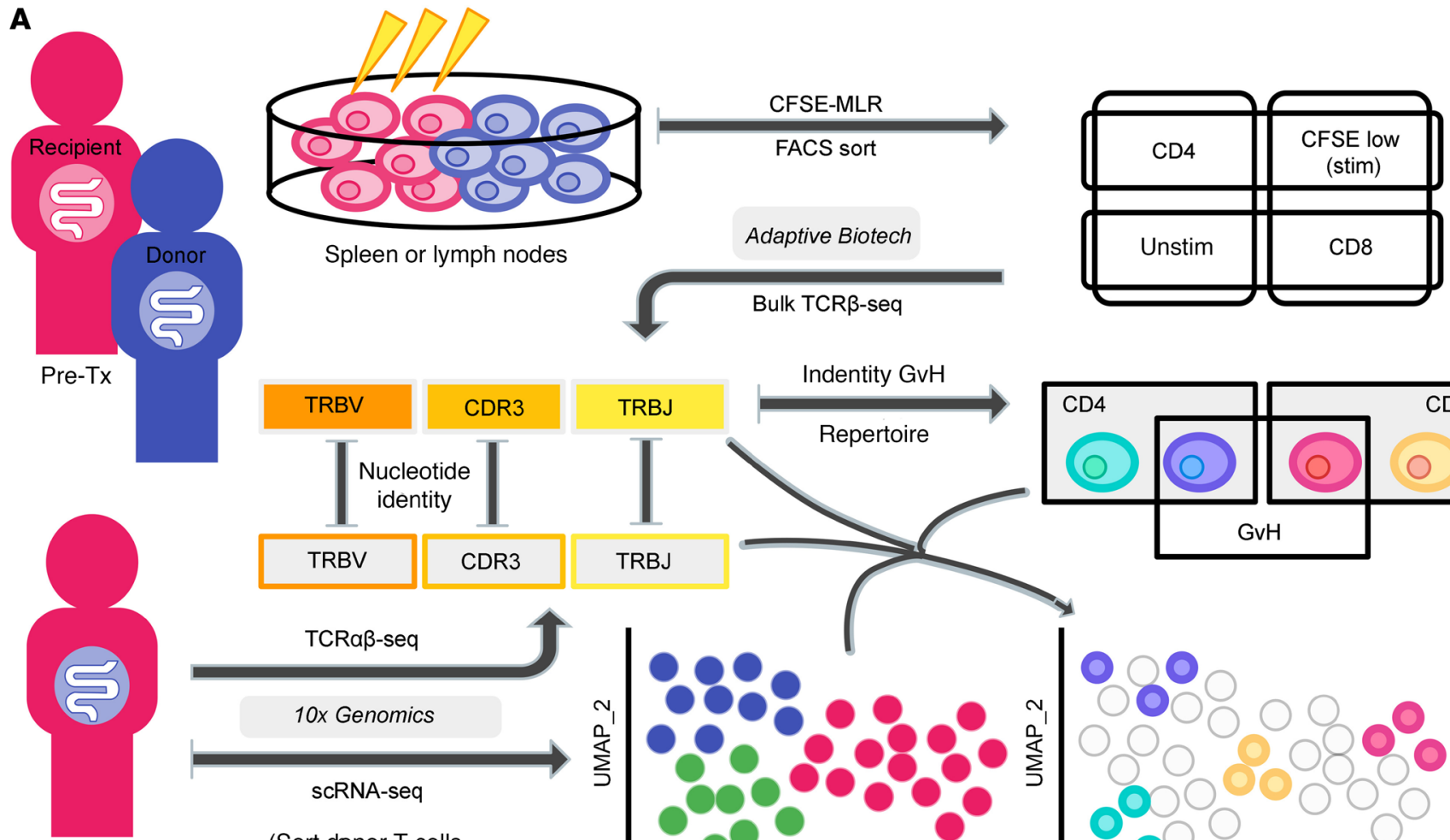

Post-tx

(Sort donor T cells from recipient bone marrow)
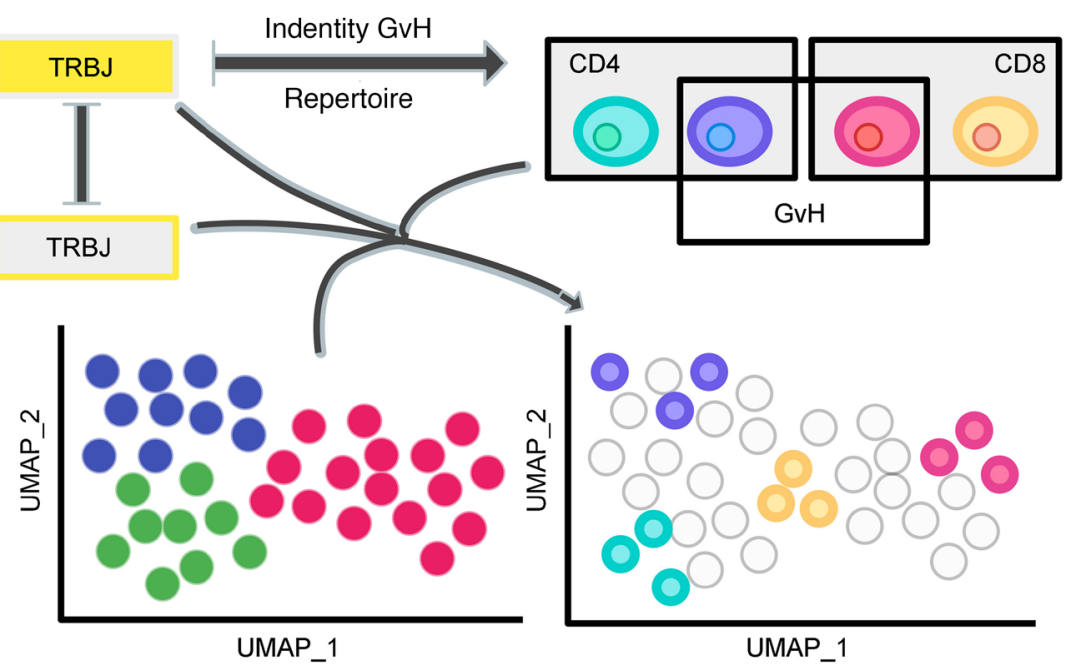

B
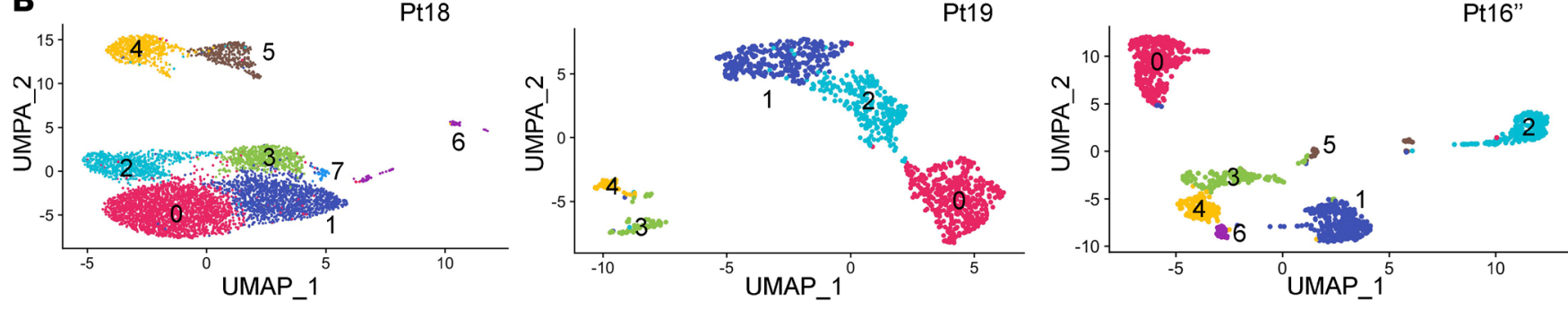

C
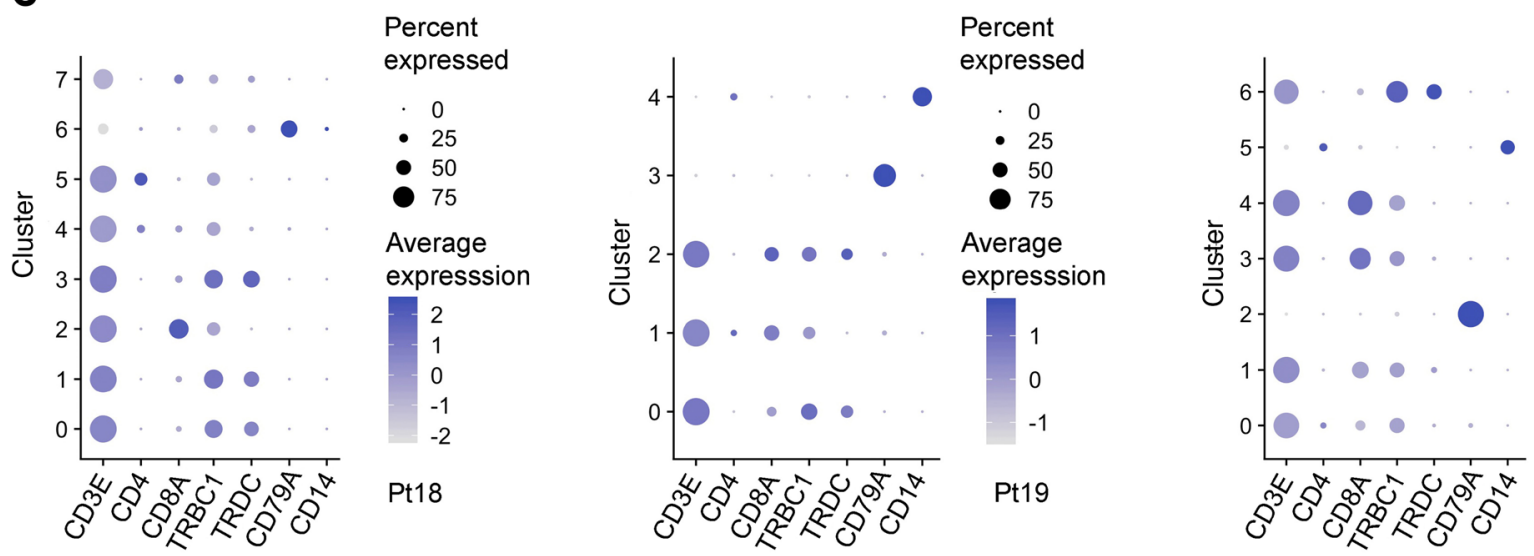

Percent expressed . 0

- 25

50

75

100

Average expresssion

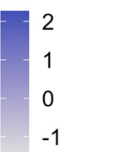

Pt16"

Figure 5. Experimental pipeline of scRNA-seq to integrate RNA profiling with T cell clonotype-defined alloreactivity and major clusters identified from BM-infiltrating donor T cells after ITx. (A) Illustration of data integration between bulk TCR- $\beta$-seq (Adaptive Biotechnologies) and scRNA-seq (10× Genomics). The latter combines 5'gene expression sequencing (5'GEX-seq) and TCR- $\alpha \beta$-seq by identifying TCR- $\beta$ chain CDR3 nucleotide + TRBV + TRB) sequences in individual cells undergoing immune profiling by transcriptional analysis. T cells are annotated as CD4 or CD8 GvH or nonGvH or as nonmappable by interrogation of the sequence sets defined as alloreactive or nonalloreactive from pre-Tx CFSE-MLRs (see Methods). (B) UMAP plots of FACS-sorted donor T cells from recipient BM from Pt18 POD357 (left panel), Pt19 POD105 (middle panel), and Pt16" POD126 (right panel). Clusters are numbered in descending order of number of cells in each. (C) Expression of T/B/monocyte lineage genes (CD3E, CD4, CD8A, TRBC1, TRDC, CD79A, and CD14) in each UMAP cluster. 
mixed chimerism induction in blood of humans after organ transplantation and its protective effects against allograft rejection, although the studies are limited by the relatively low number and heterogeneity of patients, an issue inherent to ITx. The present study also provides a rationale for initiating clinical trials of ITx combined with HSPC transplantation at the time of maximal early LGVHR.

\section{Methods}

Human subject recruitment and clinical protocols. Our cohort enrolled 24 patients, including 1 retransplant (Pt16). Given that only very limited posttransplant samples were collected from Pt8, Pt11, and Pt12, without any pretransplant specimens available to perform alloreactive clonal tracking, we excluded these 3 patients from our study. Protocol graft biopsies were obtained in the initial post-ITx period as described previously (14) and additional biopsies were performed for cause. Graft rejection was graded from negative, indeterminate, mild, and moderate to severe based on the pathologic scoring scheme, as previously reported (51). Blood samples were collected up to 4 times during the first month after Tx and thereafter at least once per month if available. Trained physicians aspirated marrow from the posterior iliac crest or another area deemed appropriate by the physician. All of the patients (Supplemental Table 1) received anti-thymocyte globulin (ATG) induction therapy (total dose: 6-10 mg/kg) followed by a maintenance regimen that included long-term tacrolimus and steroids for approximately 3 years. Tacrolimus was initiated on day 1 , the dose was adjusted to aim for a target trough level of 15 to 20 $\mathrm{ng} / \mathrm{mL}$ during the first 2 months after $\mathrm{Tx}$, and was gradually tapered down to the maintenance level of 5 to $15 \mathrm{ng} / \mathrm{mL}$ thereafter. Patients received 2 boluses of methylprednisolone on day 0 and, starting on day 1 , a dose in the range of $10 \mathrm{mg} / \mathrm{kg} /$ day followed by a taper to a maintenance dose of 3 to $5 \mathrm{mg}$ /day by 6 to 9 months, with taper off by 24 to 36 months. Allograft rejections were treated with augmented immunosuppression based on the severity of rejection. Pts 16 to 24 have been added to the recipient $\mathrm{T}$ cell repopulation study in the intestinal graft since our previous publication (14). Pt16 was retransplanted on POD786 following the first transplant. Pt16' represents the first transplant (LITx) and Pt16" represents the second transplant (MVTx) in that patient.

IEL and LPL isolations. IELs and LPLs were separated either from graft biopsy specimens or surgically obtained graft specimens at the time of stoma closure/revision, according to a protocol adapted from previous reports $(52)$ and described previously $(13,14)$. In brief, the specimens were treated for 20 minutes at $37^{\circ} \mathrm{C}$ with 2 $\mathrm{mmol} / \mathrm{L}$ dithiothreitol followed by two 30 -minute incubations with $0.5 \mathrm{mmol} / \mathrm{L}$ EDTA with continuous stirring in a water bath at $37^{\circ} \mathrm{C}$. LPLs were isolated from the remaining tissue, digested, and stirred in collagenase-containing medium (RPMI 1640, $1 \mathrm{mg} / \mathrm{mL}$ Collagenase $\mathrm{D}, 100 \mathrm{IU} / \mathrm{mL}$ penicillin-streptomycin). DNAse $(0.1 \mathrm{mg} / \mathrm{mL})$ was added to the EDTA and collagenase medium when large specimens were processed.

HLA-specific staining and cellular staining. Candidate monoclonal HLA class I allele-specific antibodies (mAbs) were screened for the ability to discriminate donor and pretransplant (pre-Tx) recipient cells, based on clinically available molecular HLA typing information. Each HLA-specific mAb was used in combination with pan-HLA-ABC antibody and quality control tested for specificity. Those that readily distinguished donor from the pre-Tx recipient PBMCs were included in lineage-specific panels of antibodies, as reported previously (12, 13). Flow cytometry antibodies used in this study are summarized as follows: HLA-ABC APC (G46-2.6, BD Biosciences, catalog 555555), HLA-ABC PE (G46-2.6, BD Biosciences, catalog 555553), HLA-ABC BV786 (G46-2.6, BD Biosciences, catalog 740982), HLA-A2/28 PE (BB7.2, BD Biosciences, catalog 558570), HLA-A2/28 FITC (One Lambda, catalog FH0037), HLA-A2/28 Biotin (One Lambda, catalog BIH0037), HLA-A9 FITC (One Lambda, catalog FH0964), HLA-A9 Biotin (One Lambda, catalog BIH0964), HLA-A3 APC (eBioscience, catalog 17-5754-42), HLA-B8 FITC (One Lambda, catalog FH0536A), HLA-B12 FITC (One Lambda, catalog FH0066), HLA-A30/31 Biotin (One Lambda, catalog BIH0067), HLA-B27 FITC (One Lambda, catalog B27F50X), CD45 V500 (HI30, BD Biosciences, catalog 560777), CD45 PE-CF594 (HI30, BD Biosciences, catalog 562279), CD3 PerCP-Cy5 (UCHT1, BD Biosciences, catalog 552852), $\gamma \delta$ TCR PE-Cy7 (immu510, Beckman Coulter, catalog PN B10247), CD4 Alexa Fluor 700 (OKT4, Tonbo Biosciences, catalog 80-0048), CD8 APC-Сy7 (SK1, BD Biosciences, catalog 557834), CD69 BV650 (FN50, BioLegend, catalog 310934), CD103 FITC (Ber-ACT8, BioLegend, catalog 350204), CD103 BV711 (Ber-ACT8, BioLegend, catalog 350222), CD45RA BV510 (HI100, BioLegend, catalog 304143), CCR7 PE-Cy7 (G043H7, BioLegend, catalog 353226), CCR7 BV421 (G043H7, BioLegend, catalog 353208), CD28 Pacific Blue (CD28.2, BioLegend, catalog 302928), CD28 PE (CD28.2, BioLegend, catalog 302908), CD34 PE (QBEnd10, Beckman Coulter, catalog IM1250U), CD25 BV421 (BC96, BioLegend, catalog 302630), Foxp3 Alexa Fluor 700 (PCH101, Thermo Fisher Scientific, catalog 56-4776-41), Streptavidin Alexa Fluor 594 (Life Technologies, catalog S32356), Streptavidin PE-Cy7 (BD Biosciences, catalog 557598), and DAPI. Data were acquired using an LSR II flow cytometer (BD Biosciences) using DIVA software. Analysis was carried out using FlowJo software (TreeStar, Inc).

CFSE-MLR and cell sorting. These assays were performed as described $(13,14,21)$. Briefly, graft-versus-host $(\mathrm{GvH})$ and hostversus-graft (HvG) mixed lymphocyte reactions (MLRs) were set up using thawed pre-Tx donor and recipient cells. A quantity of 200,000 CFSE-labeled responder cells and 200,000 violet-dye-labeled irradiated (35 Gy) stimulators were plated in each well of a round-bottom 96-well plate in MLR medium (AIM-V supplemented with 5\% AB heat-inactivated human serum, 0.01M Hepes, and $50 \mu \mathrm{m}$ 2-mercaptoethanol). MLR cultures were harvested after incubation at $37^{\circ} \mathrm{C}$ for 6 days. Cells were stained with anti-CD3, CD4, and CD8, before FACS sorting on a BD Influx cell sorter to isolate 2 discrete violet dye-negative cell populations $\left(\mathrm{CD}^{+} \mathrm{CD}^{+} \mathrm{CFSE}^{\mathrm{lo}}, \mathrm{CD}^{+} \mathrm{CD} 8^{+} \mathrm{CFSE}-\right.$ ${ }^{10}$ ), representing the $\mathrm{CD}^{+}$and $\mathrm{CD} 8^{+}$recipient-antidonor-reactive (or donor-antirecipient-reactive) $\mathrm{T}$ cells (stim). For unstimulated cell populations, pre-Tx donor and recipient cells harvested from spleen or lymph nodes (LN) were thawed and stained with anti-CD3, -CD4, and $-\mathrm{CD} 8$, and then FACS sorted into $\mathrm{CD}^{+}{ }^{+} \mathrm{CD} 4^{+}$and $\mathrm{CD} 3^{+} \mathrm{CD} 8^{+}$ populations (unstim).

TCR- $\beta$ CDR3 DNA sequencing. For Pts 4 to 21 , genomic DNA was isolated from sorted cell populations using the Qiagen DNeasy Blood and Tissue Kit. DNA was frozen at $-20^{\circ} \mathrm{C}$ and shipped on dry ice to Adaptive Biotechnologies for high-throughput TCR sequencing. For Pts 22 to 24, targeted cell populations were sorted directly into cell lysis buffer (Qiagen, catalog 158906) and shipped at room temperature to the University of Pennsylvania. Genomic DNA was isolated 


\section{A}

0 cytotoxic CD8 $\alpha \beta / \gamma \delta \mathrm{T}$

1 cytotoxic CD8 $\alpha \beta / \gamma \delta \mathrm{T}$

2 cytotoxic CD8 $\alpha \beta \mathrm{T}$

3 activated, chemotaxis related $\operatorname{CD} 8 \alpha \beta \mathrm{T} / \gamma \delta \mathrm{T}$

$4 \mathrm{Tscm} / \mathrm{Tn} / \mathrm{Tcm}$ CD4/CD8 $\alpha \beta \mathrm{T}$

5 undefined $C D 4 \alpha \beta T$

0 cytotoxic $\operatorname{CD} 8 \alpha \beta / \gamma \delta \mathrm{T}$

$1 \mathrm{Tscm} / \mathrm{Tn} / \mathrm{Tcm}$ CD4/CD8 $\alpha \beta \mathrm{T}$

2 chemotaxis related $\operatorname{CD} 8 \alpha \beta / \gamma \delta$ T

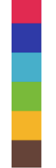

Pt18

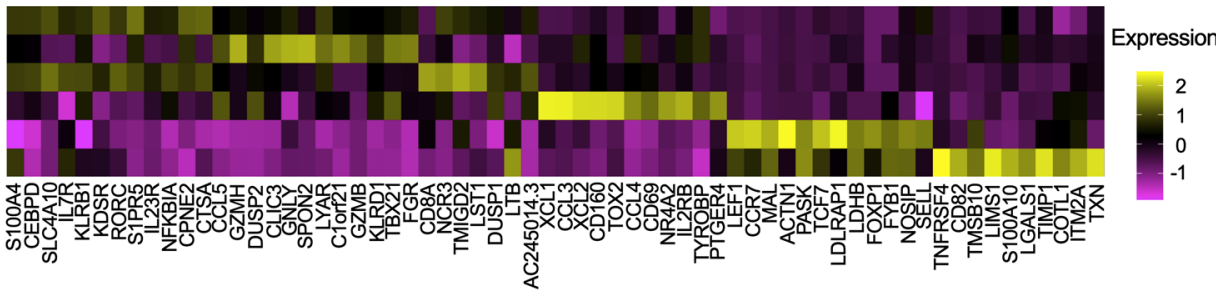

I

-
Expression

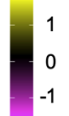

0 Tscm/Tn/Tcm CD4/CD8 $\alpha \beta$ T / $\gamma \delta$ T

1 cytotoxic CD4/CD8 $\alpha \beta \mathrm{T} / \gamma \delta \mathrm{T}$

3 cytotoxic, chemotaxis related CD8 $\alpha \beta$ T $/ \gamma \delta \mathrm{T}$

4 cytotoxic CD8 $\alpha \beta$ T

6 cytotoxic CD8 $\alpha \beta T / \gamma \delta T$

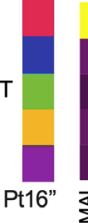

Pt16"

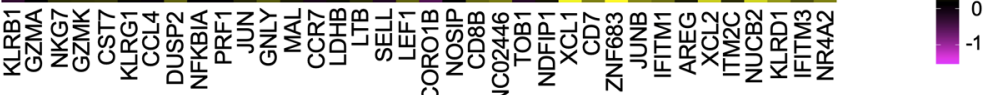

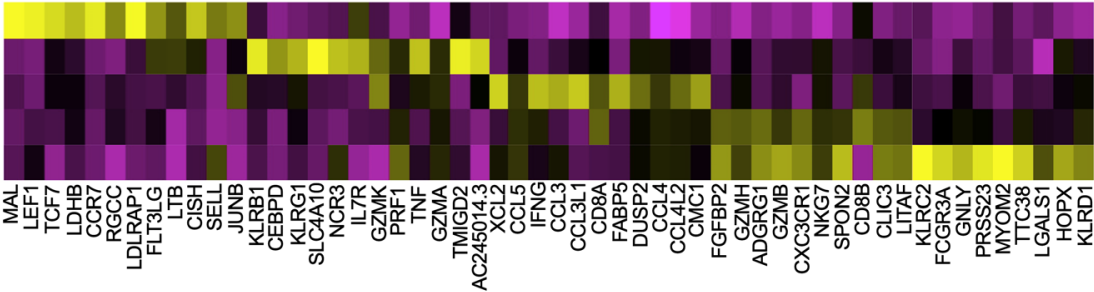

Expression

B

Shared DE genes among Pt18_cluster 0/1/2/3, Pt19_cluster 0 and Pt16"_cluster 1/3/4/6

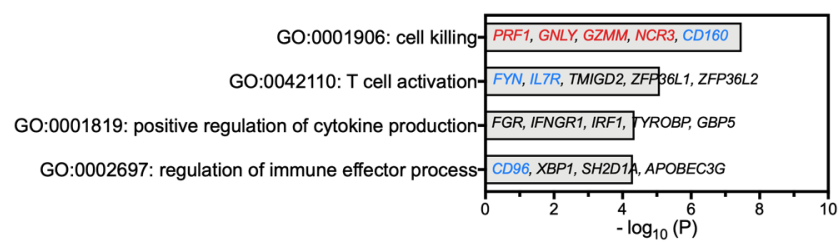

Shared DE genes among Pt18_cluster 4, Pt19_cluster 1 and Pt16"_cluster 0

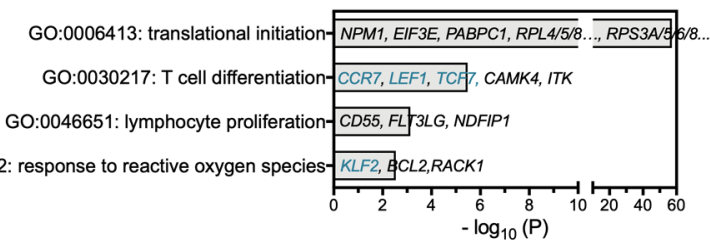

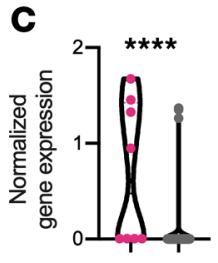

$\mathrm{TB} \times 21$

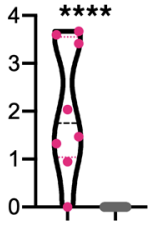

GZMB

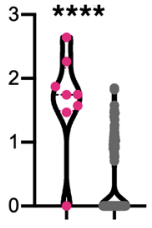

PRF1

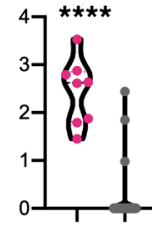

GZMA

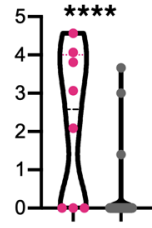

GNLY

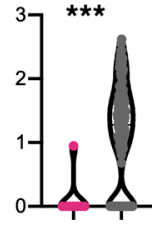

TCF7

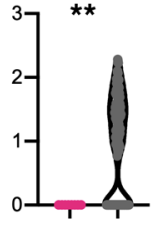

LEF1

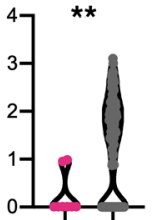

SELL

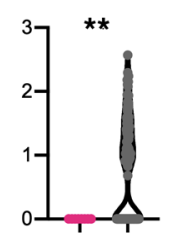

CCR7
$\mathrm{CD} 8 \mathrm{GvH}$

CD8 Tscm

Figure 6. BM-infiltrating donor T cells contain dominant clusters of cytotoxic effector T cells and undifferentiated/memory stem T cells. (A) Dominant DE genes by $\log _{2}$ fold change in major T cell clusters of each sample described in Figure 5B. FACS-sorted donor T cells were obtained from recipient BM from Pt18 POD357, Pt19 P0D105, and Pt16" P0D126. Clusters are annotated with major functional features and T cell subsets. Tscm: stem cell-like memory T cells; Tn: naive T cells; Tcm: central memory T cells. (B) GO term analysis using shared DE genes among Pt18_cluster 0/1/2/3, Pt19_cluster 0, and Pt16"_cluster 1/3/4/6 (left panel) and among Pt18_cluster 4, Pt19_cluster 1, and Pt16"_cluster 0 (right panel) are shown with up to 4 top nonredundant T cell relevant biological processes. $P<0.05\left(-\log _{10} P>1.122\right)$ is considered to be statistically significant. Representative genes related to cytotoxicity and effector T cell functions are labeled with red and light blue, respectively. Genes related to undifferentiated/memory stem T cell subsets, including Tscm, Tn, and Tcm, are labeled with teal. (C) Normalized log fold changes in expression of subset-related transcription factor genes (TBX21, TCF7, LEF1), Tscm genes (TCF7, LEF1, SELL, CCR7), and cytotoxicity genes (CZMB, PRF1, GZMA, GNLY) for CD8 GvH ( $n=8)$ and CD8 Tscm ( $n=132$ ) cells in BM-infiltrating donor T cells in Pt16" POD126. CD8 Tscm cells $(n=132)$ in cluster 0 of Pt16" expressed CD8A/CD8B but lacked CD4, TRDC, and Foxp3. Mann-Whitney $U$ test was performed. ${ }^{* * *} P<0.0001 ;{ }^{* *} P<0.001 ;{ }^{* *} P<0.01$.

from sorted cell populations using the Qiagen Gentra Puregene Kit (Qiagen, catalog 158388).

The libraries for sequencing on the Illumina MiSeq platform were prepared using a cocktail of $23 \mathrm{~V} \beta$ families from framework region 2 (FR2) forward primers, and $13 \mathrm{~J} \beta$ region reverse primers, modified from the BIOMED2 primer series (53). Primer sequences are provided in Supplemental Table 6. The PCR was performed with 2 mixes, both of which used the same $23 \mathrm{~V} \beta$ forward primers, but 2 different $\mathrm{J} \beta$ mixes. The $\mathrm{V} \beta$ and $\mathrm{J} \beta$ primers mixes were used at $0.6 \mu \mathrm{M}$ in a reaction volume of $25 \mu \mathrm{L}$ using a Multiplex PCR kit (Qiagen, catalog 158388). Amplification conditions for the PCR were as follows: primary denaturation at $95^{\circ} \mathrm{C}$ for 10 minutes, cycling at $95^{\circ} \mathrm{C}$ for 45 seconds, Ta $\left(57^{\circ} \mathrm{C}\right.$ for $\mathrm{J} \beta$ mix $1,61^{\circ} \mathrm{C}$ for $\mathrm{J} \beta$ mix 2$)$ for 90 seconds, extension at $72^{\circ} \mathrm{C}$ for 90 seconds for 35 cycles, and a final extension step at $72^{\circ} \mathrm{C}$ for 10 minutes. Amplicons were purified using the Agencourt AMPure XP beads system (Beckman Coulter, Inc) in a 1:1 ratio of beads to sam- 
ple and eluted in $40 \mu \mathrm{L}$ of TE (0.1 mM EDTA) buffer. Second-round PCRs to generate the sequencing libraries were carried out using $4 \mu \mathrm{L}$ of the first round PCR product and $2.5 \mu \mathrm{L}$ each of NexteraXT Index Primers S5XX and N7XX, using the Qiagen Multiplex PCR kit in a reaction volume of $25 \mu \mathrm{L}$. Amplification conditions for the PCR were primary denaturation at $95^{\circ} \mathrm{C}$ for 10 minutes, followed by cycling at $95^{\circ} \mathrm{C}$ for 30 seconds, $60^{\circ} \mathrm{C}$ for 30 seconds, extension at $72^{\circ} \mathrm{C}$ for 45 seconds for 8 cycles, and a final extension step at $72^{\circ} \mathrm{C}$ for 10 minutes. To confirm adequacy of amplification, aliquots of both the first- and second-round PCR products were run on agarose gels. Library quality was evaluated using Bioanalyzer 2100 (Agilent Technologies) and quantified by Qubit Fluorometric Quantitation (Thermo Fisher Scientific). A sharp single band from Bioanalyzer analysis indicated a good quality library and was used for sequencing. Readings from Qubit using the dsDNA HS (high sensitivity) assay kit (catalog Q32851) were used to calculate the molarity of the library. Libraries were then loaded onto an Illumina MiSeq in the Human Immunology Core Facility at the University of Pennsylvania. Illumina's $2 \times 300 \mathrm{bp}$ paired end kits were used for all experiments (MiSeq Reagent Kit v3, 600 cycle, catalog MS-102-3003).

TCR- $\beta$ CDR3 data processing and analysis. The TCR sequencing data for Pts 4 to 21 were retrieved from Adaptive's ImmunoSEQ software. For Pts 22 to 24, raw sequences were quality filtered as previously described $(54,55)$ and clone assemblies were processed with MiXCR (56) (v. 3.0.7) and VDJtools (57) (v1.2.1). Long CDR3 sequences, which contained nucleotides after the end of the J-gene, were truncated to adhere to IMGT numbering.

CD8 versus CD4 sorting error was corrected for by removing sequences detected in both populations at a high to low frequency ratio less than 5:1, in which case the correct subset assignment was unclear. Donor- and recipient-shared CDR3s at the nucleotide level were removed, as they could not be clearly assigned to be of either origin. After this, separate CD4 and CD8 tables containing clonal frequencies in pre-Tx unstimulated samples, $\mathrm{CFSE}^{\text {lo }}$ stimulated cells, and biopsies were compiled and renormalized. Total productive template counts (for Pts 4-21) or read counts (for Pts 22-24), a fraction of discarded sequences after removal of ambiguous clones, are summarized in Supplemental Table 2. Alloreactive clones were defined by 2 -fold or greater expansion in stimulated compared with unstimulated pre-Tx cells, and by minimum frequency of $0.001 \%$ in $\mathrm{CFSE}^{\mathrm{lo}}$ populations when using read counts, or $0.002 \%$ in $\mathrm{CFSE}^{\text {lo }}$ populations when using template counts, which serves to ensure $85 \%$ repeatability, as determined by power analysis (21). Mappable clones refers to clones that were detectable in sequenced pre-Tx spleen, lymph node, and/or MLR CFSE ${ }^{\text {lo }} \mathrm{T}$ cell populations from the donor or recipient. Cumulative frequency was calculated as a percentage of all sequences weighted by copy numbers in designated populations (Supplemental Tables 3 and 4).

scRNA-seq and data processing. scRNA-seq was performed using the $10 \times$ Genomics platform for simultaneous measurement of mRNA expression and paired V(D)J TCR- $\alpha$ and $-\beta$ sequences at the single-cell level. Briefly, iliac crest BM cells collected after Tx were ficolled to collect the mononuclear cell layer. Cells were resuspended in freezing media containing $90 \%$ human AB serum and 10\% DMSO and stored in liquid nitrogen until analysis. For scRNA-seq analysis, BM cells were thawed, washed, and sorted for viable CD45//dim donor $\mathrm{HLA}^{+} \mathrm{CD}^{+} \mathrm{T}$ cells using the BD Influx cell sorter. Cells were then mixed with $10 \times$ Chromium 5' RT reagents and loaded into a Chromium microfluidics chip and controller for droplet formation, with each productive droplet containing one single cell and one single $10 \times$ barcoded primer bead. Full-length first-strand cDNAs were synthesized in individual droplets from polyadenylated mRNAs and labeled with a unique $10 \times$ cell-bead barcode. For 5' gene expression sequencing ( $5^{\prime}$ GEX-seq), cDNAs were amplified and Illumina-compatible sequencing libraries were prepared and sequenced on an Illumina NovaSeq 6000 Sequencer. For TCR-seq, target enrichments were performed using TCR-specific outer and inner primers, followed by Illumina-compatible library preparation and sequencing on an Illumina NextSeq 550 Sequencer. FASTQ files were processed using the $10 \times$ Genomics Cell Ranger 3.1.0 with GRCh38-3.0.0 transcriptome as the reference.

scRNA-seq quality control (QC) was performed in reference to Seurat v3 pipeline $(58,59)$. Briefly, cells were filtered out if their unique feature counts were greater than $\mathrm{Q} 3+1.5{ }^{*} \mathrm{IQR}$ or less than Q1 - $1.5^{*}$ IQR (Q1: first quartile; Q3: third quartile; IQR: interquartile range, Q3-Q1). Cells were further removed if they had more than $15 \%$ mitochondrial counts. Feature expression was then normalized by the total expression within each cell, multiplying by a scale factor $(10,000)$ with a $\log _{10}$-transformation of the scaled feature expression. Two thousand highly variable features based on pipeline default were selected to calculate the principal components. UMAP plot, feature gene expression dot plot, and heatmap of differentially expressed genes were generated accordingly. Sequencing and performance metrics are summarized in Supplemental Table 7.

Our published protocol $(14,21)$ using pre-Tx MLR combined with Adaptive Biotechnology's TCR- $\beta$ bulk DNA-seq to identify GvH and nonGvH TCR- $\beta$ repertoires was applied and single-cell TCR- $\beta$ sequences of FACS-sorted donor T cells from recipient BM specimens were mapped to these pre-Tx sequence sets to allow us annotate each cell with their alloreactivity, such as CD4 or CD8 GvH or nonGvH clone or unmappable to pre-Tx donor repertoires.

Statistics. Analysis of TCR- $\beta$ repertoire bulk DNA-seq data was performed in $\mathrm{R}$ and Rstudio using our previously published scripts $(13,14)$. Analysis of scRNA-seq data was performed in R, Rstudio, and Python, with scripts partially adapted from Seurat v3 pipeline (58). EulerAPE (60) was used to generate proportional Venn diagrams. Metascape (61) was used to perform gene enrichment analysis of biological GO terms. In GO term analysis, $P$ value is the probability or chance of seeing at least $x$ number of genes out of the total $n$ genes in the list annotated to a particular GO term, given the proportion of genes in the whole genome that are annotated to that GO term. Additional statistics and figures were generated using GraphPad Prism (GraphPad Software). Student's $t$ test (2-tailed, unpaired) was used for statistical comparisons between 2 independent groups to test the means. Mann-Whitney nonparametric distribution-free $U$ test was performed to compare ranks of 2 independent samples to test the medians. Fisher's exact test was performed on binary data in unpaired samples. Kruskal-Wallis test was performed for multiple comparisons between each pair of groups when group number was greater than 2. A log-rank (Mantel-Cox) test was performed for the Kaplan-Meier plot of freedom from moderate to severe rejection of patients with $4 \%$ or more and less than $4 \%$ of donor $\mathrm{T}$ cell peak chimerism in blood. $P$ less than 0.05 was considered a statistically significant difference.

Data and materials availability. Raw TCR- $\beta$ bulk DNA-seq data for Pts 4 to 21 are freely accessible through https://doi. 
org/10.21417/JF2021JCI. Raw TCR- $\beta$ bulk DNA-seq data in FASTA format is available for Pts 22 to 24 at Sequence Read Archive (SRA: https://www.ncbi.nlm.nih.gov/sra) under BioProject accession number PRJNA578087. The code used to analyze TCR- $\beta$ bulk DNAseq data is available in the GitHub repository at https://github. com/Aleksobrad/Fu-J-et-al.-LGVHR-manuscript. The codes used to analyze $5^{\prime}$ GEX-seq and TCR- $\alpha \beta$ scRNA-seq data and integrate scRNA-seq with bulk DNA-seq by identifying nucleotide sequences of TCR- $\beta$ CDR3, $v$ and $j$, is available in the GitHub repository at https://github.com/princello/Fu-J-et-al.-LGVHR-manuscript. Raw scRNA-seq data have been deposited at SRA with BioProject accession number PRJNA610031.

Study approval. The study was approved by the Columbia University Institutional Review Board (IRB nos. AAAJ5056, AAAF2395, and AAAS7927). All subjects or legal guardians provided their written, informed consent and assent when appropriate.

\section{Author contributions}

JF, JZ, and MS designed the study. JF, JZ, BS, KF, WM, EEW, SPL, and TMS performed the experiments. JF, JZ, KF, EEW, PL, SY, KR, NMD, SR, and MM coordinated the clinical sample collection. PL, AG, and TK procured the donor organs and performed the intestinal transplant surgeries. MM performed the routine endoscopy and patient care to pediatric recipients. SR performed the routine endoscopy and patient care to adult recipients. PS performed bone marrow aspiration. AI performed the pathology review. AMR, WM, and ETLP established TCR sequencing and data analysis platform, and generated the TCR sequencing data of 3 patients. JF, JZ, BS, AO, ZW, WM, AMR, SPL, TMS, SHH, YS, ETLP, MM, TK, and MS performed data analysis. YS, AO, and ZW wrote the codes to identify and track the alloreactive clones and performed the statistical analyses. JF and MS wrote the final report. All authors contributed to the editing of the final report. All authors agreed to all the content of the submitted manuscript.

\section{Acknowledgments}

We thank Julissa Cabrera for assistance with the submission of the manuscript and Markus Mapara and Joshua Weiner for helpful review of the manuscript. We also thank Monica Velasco and Tamas Gonda for their care of intestinal transplant recipients. We thank the Flow Cytometry Core and Human Studies Core at Columbia Center for Translational Immunology (CCTI) for their excellent services. We thank Victor Xin-zheng Guo, Ya Zhang, and Yucui Zhu from the Human Immune Monitoring Core (HIMC) at Columbia University for their service and support on the scRNA-seq related study. We thank Kryscilla Yang, Christopher Parks, Rebecca Jones, Wenyu Jiao, and Alaka Gorur for their availability to assist with intestinal transplant sample processing for listed patients. This work was made possible in part by samples made available through the support of the V. Segal and S. Segal CCTI Biobank Core. We gratefully acknowledge the generosity of the donor families, our ITx patients, and their families for making this study possible. The study is part of Program Project Grant (PPG) P01 AI106697, funded by the National Institute of Allergy and Infectious Diseases (NIAID), and part of a Congressionally Directed Medical Research Program (CDMRP) Discovery Award W81XWH-20-1-0159, funded by the Department of Defense (DoD). Research reported here was performed in the CCTI Flow Cytometry Core, supported in part by the Office of the Director, National Institutes of Health (NIH) awards S10RR027050 and S10OD020056.

Address correspondence to: Megan Sykes, Columbia Center for Translational Immunology, 650 West 168th Street, Black Building 1512, Mailbox 127, New York, New York 10032, USA. Phone: 212.304.5696; Email: megan.sykes@columbia.edu.
1. Zuber J, Sykes M. Mechanisms of mixed chimerism-based transplant tolerance. Trends Immunol. 2017;38(11):829-843.

2. Kawai T, et al. HLA-mismatched renal transplantation without maintenance immunosuppression. N Engl J Med. 2008;358(4):353-361.

3. Leventhal J, et al. Chimerism and tolerance without GVHD or engraftment syndrome in HLA-mismatched combined kidney and hematopoietic stem cell transplantation. Sci Transl Med. 2012;4(124):124ra28.

4. Scandling JD, et al. Tolerance and chimerism after renal and hematopoietic-cell transplantation. N Engl J Med. 2008;358(4):362-368.

5. Kawai T, et al. Summary of the third international workshop on clinical tolerance. Am J Transplant. 2019;19(2):324-330.

6. Perri R, et al. Graft vs. host disease after liver transplantation: a new approach is needed. Liver Transpl. 2007;13(8):1092-1099.

7. Andres AM, et al. Graft-vs-host disease after small bowel transplantation in children. J Pediatr Surg. 2010;45(2):330-336.

8. Wu G, et al. Graft-versus-host disease after intestinal and multivisceral transplantation. Transplantation. 2011;91(2):219-224.

9. Sykes M, et al. Effects of T cell depletion in radiation bone marrow chimeras. II. Requirement for allogeneic $\mathrm{T}$ cells in the reconstituting bone marrow inoculum for subsequent resistance to breaking of tolerance. J Exp Med.1988;168(2):661-673.

10. Sykes M, et al. Graft-versus-host-related immunosuppression is induced in mixed chimeras by alloresponses against either host or donor lymphohematopoietic cells. J Exp Med. 1988;168(6):2391-2396.

11. Chakraverty R, et al. An inflammatory checkpoint regulates recruitment of graft-versus-host reactive T cells to peripheral tissues. JExp Med. 2006;203(8):2021-2031.

12. Zuber J, et al. Macrochimerism in intestinal transplantation: association with lower rejection rates and multivisceral transplants, without GVHD. Am J Transplant. 2015;15(10):2691-2703.

13. Fu J, et al. Human intestinal allografts contain functional hematopoietic stem and progenitor cells that are maintained by a circulating pool. Cell Stem Cell. 2019;24(2):227-239.

14. Zuber J, et al. Bidirectional intragraft alloreactivity drives the repopulation of human intestinal allografts and correlates with clinical outcome. Sci Immunol. 2016;1(4):eaah3732.

15. Smith JM, et al. OPTN/SRTR 2016 annual data report: intestine. Am J Transplant. 2018;18 Suppl 1:254-290.

16. Fishbein TM. Intestinal transplantation.
NEngl JMed. 2009;361(10):998-1008.

17. Sudan D. The current state of intestine transplantation: indications, techniques, outcomes and challenges. Am J Transplant. 2014;14(9):1976-1984.

18. Trentadue G, Dijkstra G. Current understanding of alloimmunity of the intestinal graft. Curr Opin Organ Transplant. 2015;20(3):286-294.

19. Kato T, et al. Intestinal and multivisceral transplantation in children. Ann Surg. 2006;243(6):756-764; discussion 64-66.

20. Abu-Elmagd KM, et al. Long-term survival, nutritional autonomy, and quality of life after intestinal and multivisceral transplantation. Ann Surg. 2012;256(3):494-508.

21. Morris H, et al. Tracking donor-reactive T cells: Evidence for clonal deletion in toleran kidney transplant patients. Sci Transl Med. 2015;7(272):272ra10.

22. Fujisaki J, et al. In vivo imaging of Treg cells providing immune privilege to the haematopoietic stem-cell niche. Nature. 2011;474(7350):216-219.

23. Hirata Y, et al. CD150(high) bone marrow tregs maintain hematopoietic stem cell quiescence and immune privilege via adenosine. Cell Stem Cell. 2018;22(3):445-453.

24. Abu-Elmagd K, et al. Clinical intestinal transplantation: a decade of experience at a single center. Ann Surg. 2001;234(3):404-416. 
25. Abu-Elmagd KM, et al. Five hundred intestinal and multivisceral transplantations at a single center: major advances with new challenges. Ann Surg. 2009;250(4):567-581.

26. Gaide O, et al. Common clonal origin of central and resident memory $\mathrm{T}$ cells following skin immunization. Nat Med. 2015;21(6):647-653.

27 . Beura LK, et al. T cells in nonlymphoid tissues give rise to lymph-node-resident memory $\mathrm{T}$ cells. Immunity. 2018;48(2):327-338.

28. Klicznik MM, et al. Human $\mathrm{CD} 4^{+} \mathrm{CD} 103^{+}$cutaneous resident memory $\mathrm{T}$ cells are found in the circulation of healthy individuals. Sci Immunol. 2019;4(37):eaav8995.

29. Fonseca R, et al. Developmental plasticity allows outside-in immune responses by resident memory T cells. Nat Immunol. 2020;21(4):412-421.

30. Thome JJ, et al. Early-life compartmentalization of human $\mathrm{T}$ cell differentiation and regulatory function in mucosal and lymphoid tissues. Nat Med. 2016;22(1):72-77.

31. Yolcu ES, et al. Mechanisms of tolerance induction by hematopoietic chimerism: the immune perspective. Stem Cells Transl Med. 2017;6(3):700-712.

32. Lapidot T, et al. Enhancement of T-cell-depleted bone marrow allografts in the absence of graft-versus-host disease is mediated by CD8+ CD4- and not by CD8-CD4+ thymocytes. Blood. 1992;80(9):2406-2411.

33. Reich-Zeliger S, et al. Anti-third party CD8+ CTLs as potent veto cells: coexpression of CD8 and FasL is a prerequisite. Immunity. 2000;13(4):507-515.

34. Nagai M, et al. Suppression of alloreactivity with gamma delta T-cells: relevance to increased gamma delta T-cells following bone marrow transplantation. Biomed Pharmacother. 1998;52(3):137-142.

35. Sykes M, et al. Effects of T cell depletion in radiation bone marrow chimeras. I. Evidence for a donor cell population which increases allogeneic chimerism but which lacks the potential to produce GVHD. J Immunol. 1988;141(7):2282-2288.

36. Sykes M, et al. Effects of $\mathrm{T}$ cell depletion in radiation bone marrow chimeras. III. Characterization of allogeneic bone marrow cell populations that increase allogeneic chimerism independently of graft-vs-host disease in mixed marrow recipients. Jimmunol. 1989;143(11):3503-3511.

37. Martin P. Prevention of allogeneic marrow graft rejection by donor $\mathrm{T}$ cells that do not recognize recipient alloantigens: potential role of a veto mechanism. Blood.1996;88(3):962-969.

38. Jiang Z, et al. The contribution of cytotoxic and noncytotoxic function by donor T-cells that support engraftment after allogeneic bone marrow transplantation. Biol Blood Marrow Transplant. 2002;8(11):588-596.

39. Gur $\mathrm{H}$, et al. Immune regulatory activity of CD34+ progenitor cells: evidence for a deletionbased mechanism mediated by TNF-alpha. Blood. 2005;105(6):2585-2593.

40. Mazo IB, et al. Bone marrow is a major reservoir and site of recruitment for central memory CD8+ T cells. Immunity. 2005;22(2):259-270.

41. Okhrimenko A, et al. Human memory T cells from the bone marrow are resting and maintain long-lasting systemic memory. Proc Natl Acad Sci U S A. 2014;111(25):9229-9234.

42. Di Rosa F, Gebhardt T. Bone marrow T cells and the integrated functions of recirculating and tissue-resident memory T cells. Front Immunol. 2016;7:51.

43. Noonan KA, et al. Adoptive transfer of activated marrow-infiltrating lymphocytes induces measurable antitumor immunity in the bone marrow in multiple myeloma. Sci Transl Med. 2015;7(288):288ra78.

44. Herndler-Brandstetter D, et al. The impact of aging on memory $\mathrm{T}$ cell phenotype and function in the human bone marrow. J Leukoc Biol. 2012;91(2):197-205.

45. Di Rosa F. Two niches in the bone marrow: a hypothesis on life-long $\mathrm{T}$ cell memory. Trends Immunol. 2016;37(8):503-512.

46. Baliu-Piqué M, et al. Short lifespans of memory T-cells in bone marrow, blood, and lymph nodes suggest that $\mathrm{T}$-cell memory is maintained by continuous self-renewal of recirculating cells. Front Immunol. 2018;9:2054.

47. Skirecki T, et al. Bone marrow is the preferred site of memory CD4+ T cell proliferation during recovery from sepsis. JCI Insight. 2020;5(10):134475

48. Vantourout P, Hayday A. Six-of-the-best: unique contributions of $\gamma \delta$ T cells to immunology. Nat Rev Immunol. 2013;13(2):88-100.

49. Lamb LS Jr, et al. Human gammadelta(+) T lymphocytes have in vitro graft vs. leukemia activity in the absence of an allogeneic response. Bone Marrow Transplant. 2001;27(6):601-606.

50. Schilbach KE, et al. Human gammadelta T lymphocytes exert natural and IL-2-induced cytotoxicity to neuroblastoma cells. JImmunother. 2000;23(5):536-548.

51. Remotti H, et al. Small-bowel allograft biopsies in the management of small-intestinal and multivisceral transplant recipients: histopathologic review and clinical correlations. Arch Pathol Lab Med. 2012;136(7):761-771.

52. Binda E, et al. Quantitative isolation of mouse and human intestinal intraepithelial lymphocytes by elutriation centrifugation. JImmunol Methods. 2009;344(1):26-34

53. van Dongen JJ, et al. Design and standardization of PCR primers and protocols for detection of clonal immunoglobulin and T-cell receptor gene recombinations in suspect lymphoproliferations: report of the BIOMED-2 Concerted Action BMH4CT98-3936. Leukemia. 2003;17(12):2257-2317.

54. Meng W, et al. An atlas of B-cell clonal distribution in the human body. Nat Biotechnol. 2017;35(9):879-884

55. Rosenfeld AM, et al. Computational evaluation of B-cell clone sizes in bulk populations. Front Immunol. 2018;9:1472.

56. Bolotin DA, et al. MiXCR: software for comprehensive adaptive immunity profiling. Nat Methods. 2015;12(5):380-381.

57. Shugay M, et al. VDJtools: unifying post-analysis of T cell receptor repertoires. PLoS Comput Biol. 2015;11(11):e1004503.

58. Butler A, et al. Integrating single-cell transcriptomic data across different conditions, technologies, and species. Nat Biotechnol. 2018;36(5):411-420.

59. Stuart T, et al. Comprehensive integration of single-cell data. Cell. 2019;177(7):1888-1902.

60. Micallef L, Rodgers P. eulerAPE: drawing area-proportional 3-Venn diagrams using ellipses. PLoS One. 2014;9(7):e101717.

61. Zhou Y, et al. Metascape provides a biologist-oriented resource for the analysis of systems-level datasets. Nat Commun. 2019;10(1):1523. 